\title{
The chemical composition of a regular halo globular cluster: NGC $5897^{\star, \star \star, \star \star \star}$
}

\author{
Andreas Koch ${ }^{1}$ and Andrew McWilliam ${ }^{2}$ \\ ${ }^{1}$ Zentrum für Astronomie der Universität Heidelberg, Landessternwarte, Königstuhl 12, 69117 Heidelberg, Germany \\ e-mail: akoch@lsw.uni-heidelberg.de; andy@obs.carnegiescience.edu \\ 2 Carnegie Observatories, 813 Santa Barbara St., Pasadena CA 91101, USA
}

Received 22 November 2013 / Accepted 5 March 2014

\begin{abstract}
We report for the first time on the chemical composition of the halo cluster NGC $5897\left(R_{\odot}=12.5 \mathrm{kpc}\right)$, based on chemical abundance ratios for $27 \alpha$-, iron-peak, and neutron-capture elements in seven red giants. From our high-resolution, high signal-to-noise spectra obtained with the Magellan/MIKE spectrograph, we find a mean iron abundance from the neutral species of $[\mathrm{Fe} / \mathrm{H}]=-2.04 \pm$ 0.01 (stat.) \pm 0.15 (sys.), which is more metal-poor than implied by previous photometric and low-resolution spectroscopic studies. The cluster NGC 5897 is $\alpha$-enhanced (to $0.34 \pm 0.01$ dex) and shows Fe-peak element ratios typical of other (metal-poor) halo globular clusters (GCs) with no overall, significant abundance spreads in iron or in any other heavy element. Like other GCs, NGC 5897 shows a clear $\mathrm{Na}-\mathrm{O}$ anti-correlation, where we find a prominent primordial population of stars with enhanced $\mathrm{O}$ abundances and approximately solar $\mathrm{Na} / \mathrm{Fe}$ ratios, while two stars are Na-rich, providing chemical proof of the presence of multiple populations in this cluster. Comparison of the heavy element abundances with the solar-scaled values and the metal-poor GC M15 from the literature confirms that NGC 5897 has experienced little contribution from s-process nucleosynthesis. One star of the first generation stands out in that it shows very low La and Eu abundances. Overall, NGC 5897 is a well behaved GC showing archetypical correlations and element-patterns, with little room for surprises in our data. We suggest that its lower metallicity could explain the unusually long periods of RR Lyr that were found in NGC 5897.
\end{abstract}

Key words. stars: abundances - Galaxy: abundances - Galaxy: evolution - globular clusters: individual: NGC 5897 - Galaxy: halo

\section{Introduction}

Globular clusters (GCs) are among the oldest stellar systems in the Universe and provide important information on the earliest evolutionary stages of the Galaxy. They have received much attention over the past years as they also harbor a multitude of fascinating properties. Long believed to be the classical example of simple stellar populations, it is now accepted that GCs consist of multiple stellar populations that are evident in their color-magnitude diagrams (CMDs; e.g., Piotto 2009, and references therein) and their light chemical element distributions (e.g., Gratton et al. 2012, and references therein). These are a direct consequence of the presence of at least two generations of stars, differing in age and chemical abundances, that formed within a few 100 Myr of each other. Stars of the first generations (either fast rotating massive stars, Decressin et al. 2007, or intermediate-mass asymptotic giant branch [AGB] stars, e.g., D'Ercole et al. 2008) pollute the interstellar medium (ISM) with the products of p-capture reactions, out of which the dominant, second stellar generation is born. This enrichment scenario leaves the $\alpha$ - and Fe-peak elements unaltered (as has been observed in GCs), while producing the characteristic light-element variations such as the $\mathrm{Na}-\mathrm{O}$ and $\mathrm{Mg}-\mathrm{Al}$ anti-correlations and $\mathrm{CN}$ and $\mathrm{CH}$ bimodalities.

* This paper includes data gathered with the 6.5-m Magellan Telescopes located at Las Campanas Observatory, Chile.

$\star \star$ Table 5 is available in electronic form at http://www . aanda.org

$\star \star \star$ Full Table 2 is only available at the CDS via anonymous ftp to cdsarc.u-strasbg.fr (130.79.128.5) or via

http://cdsarc.u-strasbg.fr/viz-bin/qcat?]/A+A/565/A23
Considering the broad variety in the GC properties (horizontal branch [HB] morphology, central concentration, environment, to name a few), it is then striking that several essential features are found throughout every single GC studied to date. For instance, several authors have now resorted to defining a $\mathrm{GC}$ as an object that shows a $\mathrm{Na}-\mathrm{O}$ correlation (Carretta et al. 2010). In this work, we will add the poorly-studied inner halo GC NGC 5897 to the family of typical GCs by measuring indepth its chemical composition for the first time. This will reveal if NGC 5897 also exhibits the canonical chemical abundance variations and how these could relate to the factors of environment, for example.

NGC 5897 is a metal-poor GC in the Galactic halo, located at a moderate distance from the Sun $\left(R_{\odot}=12.5 \mathrm{kpc} ; R_{\mathrm{GC}}=7 \mathrm{kpc}\right.$; Harris 2006 [2010 edition]). It shows a predominantly blue HB with a morphology that has been labeled "normal" considering its low metallicity (e.g., Clement \& Rowe 2001; Fig. 1). However, some of its other properties still do not have a satisfying explanation.

In a photometric study, Testa et al. (2001) analyzed NGC 5897 and three other GCs, which were all confirmed to be coeval and to have similar metallicity. Thus it was concluded that there must exist a very prominent, additional parameter besides age and metallicity governing the differences in their HB morphologies. This parameter was taken to be "environment", represented, for example, by the concentration or central densities of such stellar systems (Fusi-Pecci et al. 1993). In this context, NGC 5897 has a very low concentration (half-light and tidal radii are $2.1^{\prime}$ and $10.1^{\prime}$, respectively); its concentration parameter $\left(c=\log \left[r_{\mathrm{t}} / r_{\mathrm{c}}\right]\right)$ is 0.86 and only $15 \%$ of the entire known 
Milky Way GCs are less densely concentrated, where $60 \%$ of those are within the inner halo at $R_{\mathrm{GC}}<20 \mathrm{kpc}$ (Harris 1996). Additional, possible "third parameters" comprise the Helium abundance of the clusters (Gratton et al. 2010).

It is very surprising that, given the wealth of photometric data for this GC, no consensus as to its metallicity has been reached, yet: the current values for $[\mathrm{Fe} / \mathrm{H}]$ in the literature, obtained from photometry or low-resolution spectroscopy, range from -1.68 dex (Zinn \& West 1984) to -2.09 dex (Kraft \& Ivans 2003), which is by far a larger uncertainty than the possible accuracy achievable with present-day spectroscopic methods. This uncertainty would also seem to prohibit an accurate assessment even of the simplest parameters governing the HB morphology.

NGC 5897 is known to harbor several variable stars, mainly RR Lyrae. Their periods were measured to be longer than 0.6 days, which is not unusual for a low-metallicity cluster. Puzzling is, however, the unparalleled, long mean period of the RR $a b$ variables of $\left\langle P_{a b}\right\rangle>0.8 \mathrm{~d}$ (e.g., Wehlau 1990; Clement et al. 2001; Clement \& Rowe 2001), which could indicate even lower metallicities than the mean of this GC.

This work is organized as follows: In Sect. 2 we describe our target selection and data gathering; the abundance and error analyses are laid out in detail in Sects. 3, 4. All our results are presented and interpreted in Sect. 5 before summarizing our findings in Sect. 6.

\section{Observations and data reduction}

\subsection{Target selection}

Eight red giant member candidates were selected for observations from the photometric study of Testa et al. (2001) so as to cover the brightest giants while avoiding the centrally crowded regions of this cluster ${ }^{1}$. In particular, our targets are located $2.0^{\prime}$ to $5.1^{\prime}(\sim 1-2.5$ half-light radii) from the cluster center. We ensured that all targets are sufficiently unaffected by crowding (Stetson et al. 2003) and in only one case is there an estimated $\sim 0.9 \%$ flux contribution from nearby sources, while it is essentially zero for all other targets. The respective CMD including our target stars is shown in Fig. 1.

We re-derived the GC photometric parameters by fitting a grid of isochrones from the Dartmouth database (Dotter et al. 2008) to the cluster's fiducial lines by Testa et al. (2001); Fig. 1 shows the best-fit isochrone that has an age of $12.4 \mathrm{Gyr}$, an iron abundance of $-1.65 \mathrm{dex}$, and $[\alpha / \mathrm{Fe}]=0.4 \mathrm{dex}$. In the following we adopt our thus derived reddening of $E(B-V)=0.12$ and the reddening laws of Winkler (1997), as well as the best-fit distance modulus of $15.49 \mathrm{mag}$. These values are in good agreement with those derived by Testa et al. (2001). An independent comparison with isochrones from the Teramo group (Pietrinferni et al. 2004) suggests similar results of $11.5 \mathrm{Gyr},[\mathrm{Fe} / \mathrm{H}]=-1.62$, $(m-M)_{V}=15.41$, and the same reddening. In Fig. 1 we also show a metal-poor isochrone with a metallicity as found from our spectroscopy $([\mathrm{Fe} / \mathrm{H}]=-2.04)$, with similar distance modulus (15.55 mag), reddening (0.13 mag), and age (13.2 Gyr). Where possible, target IDs were taken from Sandage \& Katem (1968) to ease comparison with previous low-resolution studies.

\footnotetext{
1 Despite its loose concentration, NGC 5897's large distance renders the most central regions too dense for single-star slit spectroscopy.
}

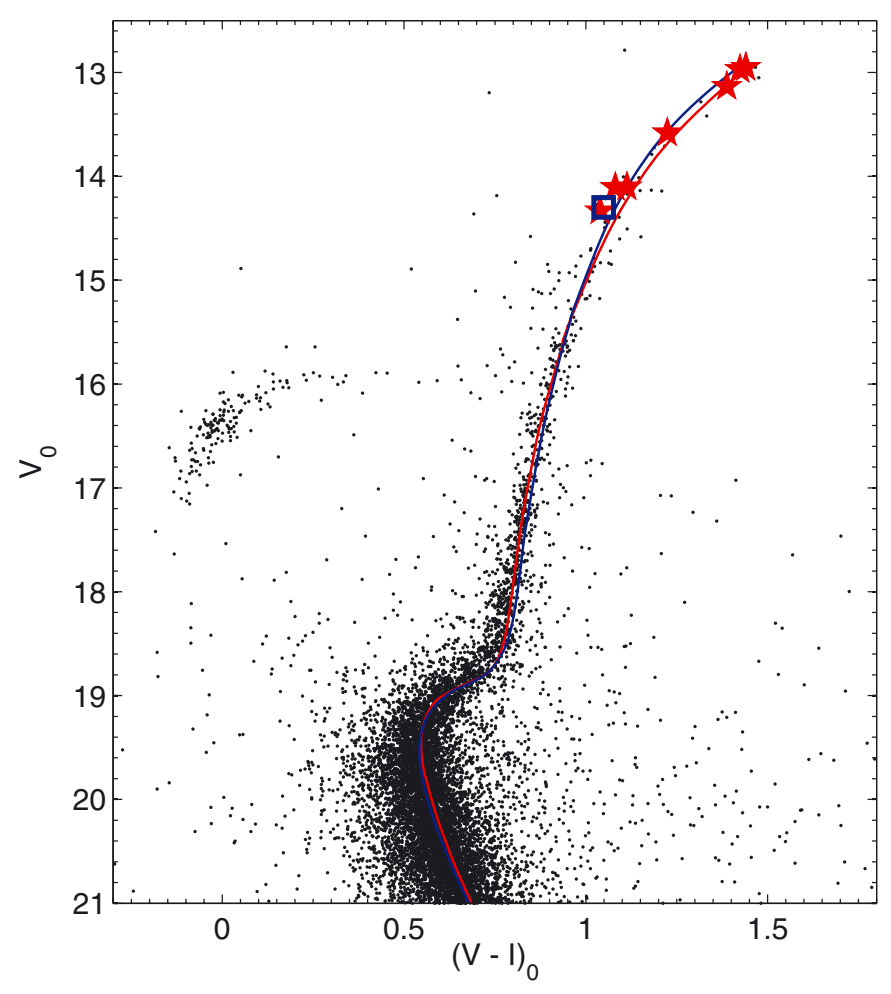

Fig. 1. CMD of NGC 5897 from Testa et al. (2001); our spectroscopic targets are highlighted as red stars. We also indicate the non-member star 11527 by a blue square. The red line is an $\alpha$-enhanced, $12.4 \mathrm{Gyr}$ isochrone (Dotter et al. 2008) for $[\mathrm{Fe} / \mathrm{H}]=-1.65$, shifted to a distance modulus of 15.49 and by a reddening vector of $E(B-V)=0.12$, while the blue curve uses our spectroscopic metallicity of $[\mathrm{Fe} / \mathrm{H}]=-2.04$ and the best distance and reddening estimates of $15.55 \mathrm{mag}$ and $0.13 \mathrm{mag}$, respectively.

\subsection{Observations}

Our observations were performed with the Magellan Inamori Kyocera Echelle (MIKE) spectrograph at the $6.5 \mathrm{~m}$ Magellan2/Clay Telescope at Las Campanas Observatory, Chile. The data were gathered over four nights in May 2013. By using a slit width of $0.5^{\prime \prime}$ and binning the CCD pixels by $2 \times 1$, we obtained a resolving power of $R \sim 40000$. Here, we used the red and blue arms of the instrument, which cover a full wavelength range of 3340-9150 $\AA$, although we primarily focused on the red wavelength region above $\sim 4900 \AA$ in our analysis. Each star was typically exposed for $0.5-2.5 \mathrm{~h}$, which we split into several exposures to facilitate cosmic ray removal. The median seeing was $0.65^{\prime \prime}$ with individual exposures reaching as high as $1^{\prime \prime}$, but notably better conditions $\left(\sim 0.4^{\prime \prime}\right)$ during the first night. The seeing variations also prompted the adaptive exposure times for stars of similar magnitudes, as seen in Table 1; e.g., for stars $\mathrm{S}-255$ and S-20 the conditions changed from $0.85^{\prime \prime}$ to $0.45^{\prime \prime}$.

The data were processed within the pipeline reduction package of Kelson (2000; 2003), which comprises flat field division, order tracing from quartz lamp flats, and wavelength calibration using built-in Th-Ar lamp exposures that were taken immediately after each science exposure. Continuum-normalization was obtained by dividing the extracted spectra by a high-order polynomial. The resulting spectra reach signal-to-noise ratios $(\mathrm{S} / \mathrm{N})$ of 90-120 per pixel as measured from the peak of the order containing $\mathrm{H} \alpha$, declining towards $\sim 65$ (35) in the bluer orders, at $4500 \AA$ (4000 $\AA$ ). An observing log is given in Table 1, which also lists the basic photometric properties of the target stars. 
Table 1. Properties of the targeted stars.

\begin{tabular}{llccccccccc}
\hline \hline ID $^{a}$ & Alt. ID & $\begin{array}{c}\alpha \\
(\mathrm{J} 2000.0)\end{array}$ & $\begin{array}{c}\delta \\
(\mathrm{J} 2000.0)\end{array}$ & $\begin{array}{c}V \\
{[\mathrm{mag}]}\end{array}$ & $\begin{array}{c}V-I \\
{[\mathrm{mag}]}\end{array}$ & $\begin{array}{c}V-K \\
{[\mathrm{mag}]}\end{array}$ & Date of obs. & $\begin{array}{c}t_{\text {exp }} \\
{[\mathrm{s}]}\end{array}$ & $\begin{array}{c}S / N^{c} \\
{\left[\mathrm{pixel}^{-1}\right]}\end{array}$ & $\begin{array}{c}v_{\mathrm{HC}} \\
{\left[\mathrm{km} \mathrm{s}^{-1}\right]}\end{array}$ \\
\hline S-255 & ESO-1702 & $15: 17: 30.07$ & $-21: 02: 08.81$ & 13.32 & 1.59 & 3.64 & 2013-May-10 & 6340 & 115 & 104.6 \\
S-20 & ESO-32 & $15: 17: 14.73$ & $-20: 56: 57.18$ & 13.34 & 1.58 & 3.59 & $2013-$ May-07 & 1800 & 110 & 100.1 \\
S-9 & ESO-3884 & $15: 17: 30.51$ & $-20: 56: 58.90$ & 13.51 & 1.54 & 3.51 & $2013-$ May-07 & 2160 & 120 & 100.9 \\
S-366 & ESO-6910 & $15: 17: 14.06$ & $-21: 04: 19.72$ & 13.95 & 1.38 & 3.10 & 2013-May-07 & 3600 & 105 & 101.8 \\
S-138 & ESO-4959 & $15: 17: 39.38$ & $-20: 59: 53.25$ & 14.48 & 1.27 & 3.06 & 2013-May-07, 08 & 8100 & 120 & 101.1 \\
S-290 & ESO-5660 & $15: 17: 09.41$ & $-21: 01: 46.98$ & 14.48 & 1.23 & 2.89 & 2013-May-10 & 6600 & 100 & 102.2 \\
S-364 & ESO-1974 & $15: 17: 21.49$ & $-21: 04: 40.91$ & 14.71 & 1.19 & 2.83 & 2013-May-11 & 5400 & 90 & 103.3 \\
\hline$\ldots$ & ESO-4678 & $15: 17: 45.47$ & $-21: 01: 54.93$ & 14.67 & 1.20 & 2.73 & 2013-May-10,11 & 5400 & 100 & -52.7 \\
\hline
\end{tabular}

Notes. ${ }^{(a)}$ Identifications from Sandage \& Katem (1968). ${ }^{(b)}$ ID from Testa et al. (2001). ${ }^{(c)}$ Measured in the order containing H $\alpha .{ }^{(d)}$ Variable star $\equiv$ V-5 (Sandage \& Katem 1968). ${ }^{(e)}$ Non-member.

\subsection{Radial velocities}

We measured radial velocities of the targets by cross-correlating the spectra in the range 5500-6500 $\AA$ against a synthetic spectrum of a red giant with stellar parameters representative of the upper RGB of NGC 5897, as expected for our targets. This yielded typical uncertainties of $\sim 0.2 \mathrm{~km} \mathrm{~s}^{-1}$. Five of our targets were included in the low-resolution sample of Geisler et al. (1995), who measured radial velocities and metallicities from the near-infrared calcium triplet $(\mathrm{CaT})$. On average, we find an excellent agreement with the values of Geisler et al. (1995); individual differences of up to $2 \mathrm{~km} \mathrm{~s}^{-1}$ could indicate possible binarity of some targets, but since our prime motive is not a detailed kinematic study of this GC, we did not investigate the velocities further. Likewise, for the two stars we have in common with the CaT sample of Rutledge et al. (1997) we measured velocities higher by $\sim 4 \mathrm{~km} \mathrm{~s}^{-1}$.

One of our targets, ESO-4678, turned out to be a nonmember with a highly deviant radial velocity and different metallicity. While listed in Table 1 for completeness, we ignore this object for the remainder of this work. All other stars are confirmed cluster members.

\section{Abundance analysis}

We derived chemical element abundances through a standard, absolute analysis using equivalent widths (EWs) that closely follows the procedures used in our previous works (e.g., Koch et al. 2009; Kacharov et al. 2013). All analyses employed the 2010 version of the stellar abundance code MOOG (Sneden 1973).

\subsection{Line list}

The line list for this work builds on that used in Koch \& Côté (2010), which, in turn, was assembled from various sources (see references therein). Additional transitions for several heavy elements (Zr, La, Ce, Dy) were extracted from Shetrone et al. (2003), Sadakane et al. (2004), and Yong et al. (2005).

The EWs were measured by fitting a Gaussian profile to the absorption lines using IRAF's splot. The final line list for this absolute analysis is provided in Table 2. Individual elements and transitions will be discussed further in Sect. 5.

The effects of hyperfine structure were considered for lines of the odd- $Z$ elements Mn I, Cu I, Ba II, La II, and Eu II, using data from McWilliam et al. (1995), as well as the splitting for Sc II, V I, and Co I, albeit very small (typically $\lesssim 0.04$ dex).
Finally, the abundance results were placed on the solar, photospheric scale of Asplund et al. (2009).

\subsection{Stellar parameters and atmospheres}

As before, we interpolated the model atmospheres from Kurucz's ${ }^{2}$ grid of one-dimensional 72-layer, plane-parallel, lineblanketed models without convective overshoot, assuming local thermodynamic equilibrium (LTE) for all species. This model grid incorporates the $\alpha$-enhanced opacity distribution functions, AODFNEW (Castelli \& Kurucz 2003) ${ }^{3}$. This is a justified assumption, since the majority of the metal-poor Galactic halo GCs (and field stars) are enhanced in the $\alpha$-elements by $\approx+0.4$ dex. We expect NGC 5897 to also follow this trend, as was confirmed in retrospective by our measurements of an elevated $[\alpha / \mathrm{Fe}]($ Sect. 5.3).

\subsubsection{Spectroscopic temperatures}

We derived spectroscopic temperatures by forcing excitation equilibrium, i.e., by removing the trend in the abundance from the Fe I lines with excitation potential. In this and the following steps we restricted the analysis to moderately strong lines that meet $-5.5 \leq \log (E W / \lambda) \leq-4.5$. The spectroscopic $T_{\text {eff }}$ is thus typically precise to within $\pm 100 \mathrm{~K}$, based on the range of reasonable slopes. While deriving the GC abundance distribution upon relying on the spectroscopic values, we noted strong trends of some abundances with temperature. Particularly strongly affected were Fe I and Fe II, and accordingly the ensuing abundance ratios $[\mathrm{X} / \mathrm{Fe}]$ of many elements. Thus the Pearson correlation coefficient, $r$, is $-0.76 \pm 0.15$ for $\mathrm{Fe} \mathrm{I}$ and $-0.89 \pm 0.14$ for Fe II.

\subsubsection{Photometric temperatures}

Next, photometric temperatures were gauged from stellar $(V-I)$ and $(V-K)$ colors; to this end, we applied the temperature-color calibrations of Ramírez \& Meléndez (2005) to the photometry of Testa et al. (2001), to which we added the $K$-band magnitudes from the 2MASS (Cutri et al. 2003). For this, a metallicity of -1.7 dex from previous photometric and CaT-studies was used as an initial estimate in the calibrations. From this, we found that $T_{\text {eff }}(V-K)$ is cooler than $T_{\text {eff }}(V-I)$ by $66 \mathrm{~K}$ $(1 \sigma$-scatter of $41 \mathrm{~K})$, but we note that systematic trends in the

\footnotetext{
2 http://cfaku5.cfa.harvard.edu/grids.html

3 See http://wwwuser.oat.ts.astro.it/castelli
} 
Table 2. Line list.

\begin{tabular}{|c|c|c|c|c|c|c|c|c|c|c|}
\hline \multirow{2}{*}{ Element } & \multirow{2}{*}{$\begin{array}{c}\lambda \\
{[\AA]}\end{array}$} & \multirow{2}{*}{$\begin{array}{l}\text { E.P. } \\
{[\mathrm{eV}]}\end{array}$} & \multirow{2}{*}{$\log g f$} & \multicolumn{7}{|c|}{$E W[\mathrm{~m} \AA]$} \\
\hline & & & & S-255 & S-20 & S-9 & S-366 & S-138 & S-290 & S-364 \\
\hline [O I] & 5577.34 & 1.97 & -8.204 & 5 & & 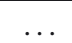 & & . & 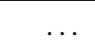 & 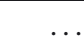 \\
\hline$[\mathrm{O} \mathrm{I}]$ & 6300.31 & 0.00 & -9.819 & 45 & 32 & 40 & 9 & 23 & 21 & 12 \\
\hline [O I] & 6363.78 & 0.02 & -10.303 & 16 & 19 & 11 & 4 & 7 & 5 & 6 \\
\hline $\mathrm{Na} \mathrm{I}$ & 5682.63 & 2.10 & -0.700 & 18 & 48 & 16 & 38 & 12 & 14 & 14 \\
\hline $\mathrm{Na} \mathrm{I}$ & 5688.20 & 2.10 & -0.460 & 60 & 73 & 30 & 64 & 11 & 30 & 21 \\
\hline $\mathrm{Na} \mathrm{I}$ & 6154.23 & 2.10 & -1.560 & 7 & 11 & $\ldots$ & 7 & $\ldots$ & 3 & $\ldots$ \\
\hline $\mathrm{Na} \mathrm{I}$ & 6160.75 & 2.10 & -1.260 & 14 & 24 & $\ldots$ & 12 & 5 & 5 & 6 \\
\hline Mg I & 5528.42 & 4.35 & -0.481 & 167 & 160 & 161 & 135 & 130 & 129 & 120 \\
\hline $\mathrm{Mg} \mathrm{I}$ & 5711.09 & 4.33 & -1.660 & 59 & 56 & 61 & 40 & 38 & 35 & 33 \\
\hline Mg I & 7387.69 & 5.75 & -1.020 & 14 & 9 & 15 & 11 & 18 & 13 & 10 \\
\hline
\end{tabular}

Notes. Table 2 is available in its entirety in electronic form at the CDS.

Table 3. Atmospheric parameters.

\begin{tabular}{|c|c|c|c|c|c|c|}
\hline \multirow{2}{*}{ ID } & \multicolumn{4}{|c|}{$T_{\text {eff }}[\mathrm{K}]$} & \multirow{2}{*}{$\log g$} & \multirow{2}{*}{$\begin{array}{c}\xi \\
{\left[\mathrm{km} \mathrm{s}^{-1}\right.}\end{array}$} \\
\hline & (spec.) & $(V-K)$ & $(V-I)$ & (phot.) & & \\
\hline S-255 & 4000 & 4077 & 4174 & 4126 & 0.44 & 2.60 \\
\hline S-20 & 4025 & 4101 & 4185 & 4143 & 0.46 & 2.30 \\
\hline S-9 & 4125 & 4146 & 4218 & 4182 & 0.56 & 2.25 \\
\hline S-366 & 4300 & 4400 & 4394 & 4397 & 0.92 & 2.00 \\
\hline S-138 & 4370 & 4433 & 4551 & 4492 & 1.19 & 1.90 \\
\hline S-290 & 4400 & 4566 & 4596 & 4581 & 1.25 & 2.00 \\
\hline S-364 & 4450 & 4607 & 4670 & 4639 & 1.38 & 2.05 \\
\hline
\end{tabular}

$(V-K)$ temperature scale have been reported before (Fabbian et al. 2009; Kacharov et al. 2013). Overall, our mean photometric values are higher than the spectroscopic temperatures by $128 \pm 17 \mathrm{~K}$ on average. However, most importantly, there is no systematic trend in the Fe abundance from the neutral species with photometric temperature $(r=-0.19 \pm 0.22)$, while the trend in the ionized species remains. The exact same behavior is seen, e.g., in the data of Yong et al. (2003; their Fig. 3) and they argue that a combination of stellar parameter changes can account for these trends rather than resorting to higher-order effects like NLTE corrections.

By using the photometric temperature scale instead of the spectroscopic one, as requested by the referee, we alleviate the mean ionization imbalance (Sect. 3.2.4) from $[\mathrm{Fe} \mathrm{I} / \mathrm{Fe} \mathrm{II}]=$ -0.34 to -0.12 dex. Thus, we continued the analyses by using the mean of both color indices as photometric $T_{\text {eff }}$ (labeled "phot." below) in our atmospheres. The atmospheric parameters of the red giant sample are listed in Table 3.

\subsubsection{Microturbulence}

We determined microturbulent velocities, $\xi$, from the plot of abundances versus reduced width, $\log (E W / \lambda)$, of neutral iron lines. The range of slopes in this fit indicates typical uncertainties in $\xi$ of $0.2 \mathrm{~km} \mathrm{~s}^{-1}$ at most. We show in Fig. 2 the resulting excitation plot and EW plot and the ensuing, near-flat trends in those plots.

\subsubsection{Ionization (non-)equilibrium}

Surface gravities were derived from basic stellar structure considerations (e.g., Eq. (1) in Koch \& McWilliam 2008) using the photometric $T_{\text {eff }}$, the $V$-band photometry of Testa et al. (2001),
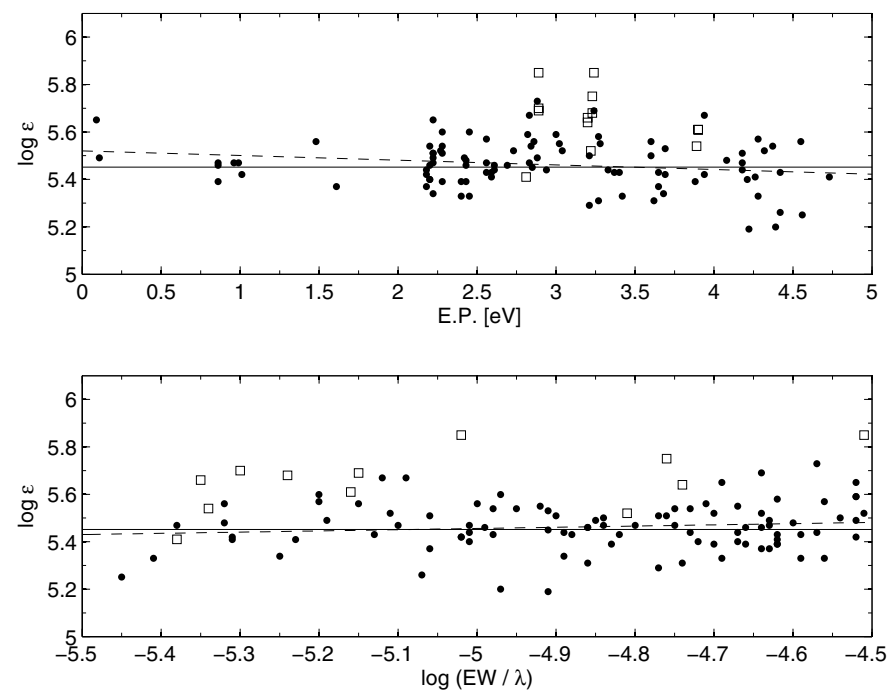

Fig. 2. Excitation (top panel) and equivalent width (bottom panel) plots for neutral (points) and ionized (open squares) Fe lines in star S-9. The solid and dashed lines indicate the mean abundance from the Fe 1 lines and the linear regression, respectively.

and adopting a distance to NGC 5897 of $12.5 \mathrm{kpc}$ (Harris 2006; Testa et al. 2001), which was found to yield a satisfactory fit to the CMD in Fig. 1. Comparison of the colors and magnitudes of the target stars with the aforementioned isochrones indicates an average stellar mass of $0.79 M_{\odot}$ for the red giants, which entered in the $\log g$ determinations.

Uncertainties in the temperatures $( \pm 130 \mathrm{~K})$, distance $( \pm 0.5 \mathrm{kpc})$, photometry and reddening $( \pm 0.05 \mathrm{mag})$, stellar mass $\left( \pm 0.1 M_{\odot}\right)$, and input metallicity $( \pm 0.1 \mathrm{dex})$ imply an average gravity error of $\sim 0.15$ dex. Ionization equilibrium is not fulfilled in our stars and we find a mean difference in $[\mathrm{Fe} \mathrm{I} / \mathrm{Fe}$ II] of $-0.12 \pm 0.03$ dex, while $\varepsilon$ (Ti I $)-\varepsilon$ (Ti II) is $-0.26 \pm 0.04$ dex. These values are very similar to those we found in the comparably metal-poor GC NGC 6397 (Koch \& McWilliam 2011). To test whether the Ti imbalance could be due to systematic errors in the $g f$-scales, we also employed the most recent laboratory oscillator strengths for Ti I (Lawler et al. 2013) and Ti II (Wood et al. 2013), which are available for a subset of our line list. These values even deteriorate the non-equilibrium in Ti I/II so that we discard this systematic effect as an explanation. Problems with NLTE and ionization equilibrium for Ti have long been suspected in RGB stars (e.g., Ruland et al. 1980; cf. Fulbright et al. 2007). 
Table 4. Error analysis for the red giants S-255 and S-364.

\begin{tabular}{|c|c|c|c|c|c|c|c|c|c|c|c|c|}
\hline \multirow[t]{2}{*}{ Ion } & $\begin{array}{c}\Delta T_{\text {eff }} \\
\pm 100 \mathrm{~K}\end{array}$ & $\begin{array}{c}\Delta \log g \\
\pm 0.2 \mathrm{dex}\end{array}$ & $\begin{array}{c}\Delta[\mathrm{M} / \mathrm{H}] \\
\pm 0.1 \mathrm{dex}\end{array}$ & $\begin{array}{c}\Delta \xi \\
\pm 0.2 \mathrm{~km} \mathrm{~s}^{-1}\end{array}$ & ODF & $\sigma_{\text {tot }}$ & $\begin{array}{c}\Delta T_{\text {eff }} \\
\pm 100 \mathrm{~K}\end{array}$ & $\begin{array}{c}\Delta \log g \\
\pm 0.2 \mathrm{dex}\end{array}$ & $\begin{array}{c}\Delta[\mathrm{M} / \mathrm{H}] \\
\pm 0.1 \mathrm{dex}\end{array}$ & $\begin{array}{c}\Delta \xi \\
\pm 0.2 \mathrm{~km} \mathrm{~s}^{-1}\end{array}$ & ODF & $\sigma_{\text {tot }}$ \\
\hline & \multicolumn{6}{|c|}{ S-255 } & \multicolumn{6}{|c|}{$\mathrm{S}-364$} \\
\hline $\mathrm{Fe} I$ & $\begin{array}{l}+0.12 \\
-0.08\end{array}$ & $\mp 0.01$ & $\mp 0.01$ & $\mp 0.09$ & 0.01 & 0.13 & \pm 0.15 & $\mp 0.02$ & $\mp 0.01$ & $\mp 0.07$ & 0.04 & 0.17 \\
\hline Fe II & $\begin{array}{l}+0.11 \\
-0.21\end{array}$ & $\begin{array}{l}+0.03 \\
-0.05\end{array}$ & \pm 0.02 & $\mp 0.05$ & -0.06 & 0.17 & $\begin{array}{l}+0.05 \\
-0.07\end{array}$ & $\begin{array}{l}+0.06 \\
-0.08\end{array}$ & \pm 0.02 & $\mp 0.04$ & -0.05 & 0.10 \\
\hline O I & $\mp 0.01$ & \pm 0.06 & \pm 0.03 & $<0.01$ & -0.08 & 0.07 & \pm 0.04 & \pm 0.08 & \pm 0.02 & $<0.01$ & -0.07 & 0.09 \\
\hline $\mathrm{Na} I$ & \pm 0.10 & $\begin{array}{l}+0.03 \\
-0.04\end{array}$ & $\mp 0.02$ & $\mp 0.01$ & 0.06 & 0.11 & \pm 0.08 & $\mp 0.02$ & $\mp 0.01$ & $<0.01$ & 0.03 & 0.08 \\
\hline $\operatorname{Mg} I$ & $\begin{array}{l}+0.08 \\
-0.05\end{array}$ & $\mp 0.03$ & $\mp 0.02$ & $\mp 0.05$ & 0.04 & 0.09 & \pm 0.07 & $\mp 0.02$ & $\mp 0.01$ & $\mp 0.03$ & 0.03 & 0.08 \\
\hline $\mathrm{Al} \mathrm{I}$ & \pm 0.09 & $\mp 0.03$ & $\mp 0.01$ & $<0.01$ & 0.04 & 0.10 & \pm 0.08 & $\mp 0.01$ & $<0.01$ & $<0.01$ & 0.02 & 0.08 \\
\hline Si I & $\begin{array}{l}+0.01 \\
-0.08\end{array}$ & \pm 0.01 & \pm 0.01 & $<0.01$ & -0.02 & 0.05 & $\begin{array}{l}+0.03 \\
-0.01\end{array}$ & \pm 0.01 & $<0.01$ & $<0.01$ & 0.00 & 0.02 \\
\hline K I & $\begin{array}{l}+0.19 \\
-0.22\end{array}$ & $\begin{array}{l}+0.02 \\
-0.00\end{array}$ & $\mp 0.01$ & $\begin{array}{l}+0.08 \\
-0.10\end{array}$ & -0.00 & 0.23 & \pm 0.15 & $\mp 0.02$ & $\mp 0.02$ & $\mp 0.10$ & 0.04 & 0.18 \\
\hline $\mathrm{Ca} \mathrm{I}$ & \pm 0.15 & $\mp 0.03$ & $\mp 0.02$ & $\mp 0.05$ & 0.05 & 0.17 & \pm 0.11 & $\mp 0.02$ & $\mp 0.01$ & $\mp 0.04$ & 0.04 & 0.12 \\
\hline Sc II & $\begin{array}{l}+0.01 \\
-0.05\end{array}$ & \pm 0.04 & \pm 0.02 & $\mp 0.05$ & -0.05 & 0.07 & $\mp 0.01$ & \pm 0.06 & \pm 0.02 & $\mp 0.03$ & -0.06 & 0.08 \\
\hline Ti I & $\begin{array}{l}-0.03 \\
+0.23 \\
-0.27\end{array}$ & $\mp 0.01$ & $\mp 0.01$ & $\mp 0.09$ & -0.01 & 0.27 & ${ }_{-0.22}^{+0.20}$ & $\mp 0.03$ & $\mp 0.02$ & $\mp 0.04$ & 0.05 & 0.22 \\
\hline Ti II & $\begin{array}{l}+0.02 \\
-0.05\end{array}$ & \pm 0.01 & $<0.01$ & $\mp 0.08$ & -0.03 & 0.09 & $\begin{array}{l}{ }_{-0.00}^{+0.00} \\
-0.02\end{array}$ & \pm 0.06 & \pm 0.02 & $\begin{array}{l}+0.06 \\
-0.09\end{array}$ & -0.05 & 0.10 \\
\hline$V_{\text {I }}$ & $\begin{array}{l}+0.23 \\
-0.26\end{array}$ & $\mp 0.01$ & $\mp 0.01$ & $\mp 0.01$ & 0.01 & 0.25 & $\begin{array}{l}+0.20 \\
-0.22\end{array}$ & $\mp 0.03$ & $\mp 0.02$ & $\mp 0.01$ & 0.05 & 0.21 \\
\hline $\mathrm{Cr} \mathrm{I}$ & $\begin{array}{l}+0.22 \\
-0.25\end{array}$ & $\mp 0.02$ & $\mp 0.02$ & $\begin{array}{l}+0.10 \\
-0.12\end{array}$ & 0.02 & 0.26 & \pm 0.19 & $\mp 0.03$ & $\mp 0.02$ & $\mp 0.07$ & 0.06 & 0.20 \\
\hline Mn I & $\begin{array}{l}+0.18 \\
-0.16\end{array}$ & $\mp 0.01$ & $\mp 0.01$ & $\mp 0.03$ & 0.02 & 0.17 & \pm 0.17 & $\mp 0.02$ & $\mp 0.01$ & $<0.01$ & 0.04 & 0.17 \\
\hline Co I & $\begin{array}{l}+0.13 \\
-0.09\end{array}$ & $\mp 0.01$ & $<0.01$ & $\mp 0.02$ & 0.01 & 0.11 & \pm 0.16 & $\begin{array}{l}+0.00 \\
-0.02\end{array}$ & $\mp 0.01$ & $<0.01$ & 0.04 & 0.16 \\
\hline Ni I & $\begin{array}{l}{ }_{-0.04}^{+0.09} \\
-0.04\end{array}$ & \pm 0.01 & \pm 0.01 & $\mp 0.04$ & -0.01 & 0.08 & \pm 0.12 & $\mp 0.01$ & $\mp 0.01$ & $\mp 0.02$ & 0.03 & 0.13 \\
\hline $\mathrm{Cu} \mathrm{I}$ & $\begin{array}{l}{ }_{-0.12}^{+0.12} \\
-0.08\end{array}$ & $\mp 0.01$ & $<0.01$ & $\mp 0.02$ & 0.01 & 0.10 & \pm 0.16 & $\mp 0.02$ & $\mp 0.01$ & $\mp 0.01$ & 0.04 & 0.16 \\
\hline Y II & $\begin{array}{l}+0.00 \\
{ }_{-0.02}\end{array}$ & \pm 0.02 & \pm 0.01 & $\mp 0.04$ & -0.03 & 0.05 & \pm 0.01 & \pm 0.06 & \pm 0.02 & $\mp 0.04$ & -0.05 & 0.07 \\
\hline Ba II & $\begin{array}{l}-0.02 \\
+0.05 \\
-0.02\end{array}$ & \pm 0.06 & \pm 0.02 & $\begin{array}{l}+0.13 \\
-0.16\end{array}$ & -0.09 & 0.16 & $\begin{array}{l}+0.05 \\
{ }_{-0.03}\end{array}$ & \pm 0.07 & \pm 0.02 & $\begin{array}{l}+0.14 \\
{ }_{-0.16}\end{array}$ & -0.07 & 0.17 \\
\hline La II & $\begin{array}{l}{ }_{-0.03}^{+0.03} \\
-0.02\end{array}$ & \pm 0.06 & \pm 0.03 & $\mp 0.01$ & -0.08 & 0.08 & \pm 0.04 & \pm 0.07 & \pm 0.02 & $<0.01$ & -0.06 & 0.08 \\
\hline Ce II & $\mp 0.01$ & \pm 0.05 & \pm 0.02 & $<0.01$ & -0.06 & 0.05 & \pm 0.03 & \pm 0.07 & \pm 0.02 & $<0.01$ & -0.05 & 0.08 \\
\hline Pr II & $\begin{array}{l}{ }_{-0.01}^{+0.04} \\
{ }_{0}\end{array}$ & \pm 0.05 & \pm 0.03 & $\mp 0.01$ & -0.06 & 0.07 & \pm 0.04 & \pm 0.07 & \pm 0.02 & $\mp 0.01$ & -0.06 & 0.09 \\
\hline $\mathrm{Nd}$ II & $\mp 0.02$ & \pm 0.04 & \pm 0.02 & $\mp 0.02$ & -0.05 & 0.05 & $\begin{array}{l}{ }_{-0.02}^{+0.04} \\
-0.0\end{array}$ & \pm 0.07 & \pm 0.03 & $\mp 0.02$ & -0.05 & 0.08 \\
\hline Sm II & $\begin{array}{l}{ }_{-0.02}^{+0.03} \\
\text {. }\end{array}$ & \pm 0.02 & \pm 0.01 & $\mp 0.02$ & -0.04 & 0.04 & \pm 0.04 & \pm 0.06 & \pm 0.02 & $\mp 0.01$ & -0.05 & 0.08 \\
\hline Eu II & $\begin{array}{l}+0.00 \\
-0.04\end{array}$ & \pm 0.07 & $\begin{array}{l}+0.04 \\
-0.02\end{array}$ & $\mp 0.01$ & -0.08 & 0.08 & $\mp 0.01$ & \pm 0.08 & \pm 0.03 & $\mp 0.01$ & -0.07 & 0.08 \\
\hline Gd II & $\mp 0.01$ & $<0.01$ & $<0.01$ & $\mp 0.01$ & $<0.01$ & 0.02 & \pm 0.03 & \pm 0.05 & \pm 0.02 & $\mp 0.01$ & -0.04 & 0.06 \\
\hline
\end{tabular}

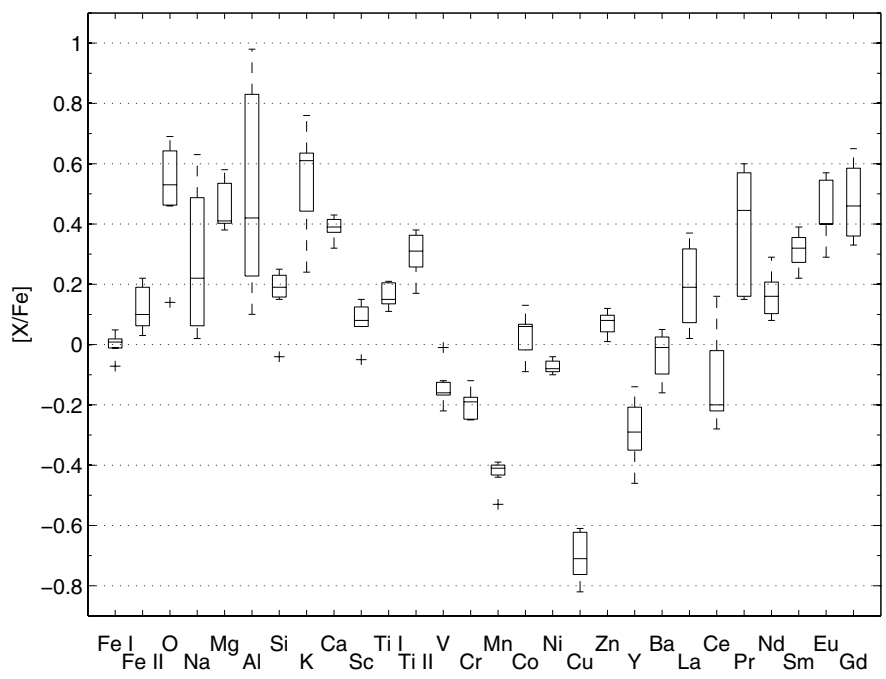

Fig. 3. Boxplot showing the median (solid horizontal lines) and interquartile $(25-75 \%)$ ranges of the chemical abundance ratios in NGC 5897 with the full abundance coverage depicted by the dashed error bars. Plus symbols designate $1 \sigma$ outliers. All values are shown relative to the respective ionization stage, except for Fe I (relative to the cluster mean), Fe II (relative to Fe I), and O I (relative to Fe II.)
We did not attempt to further enforce this equilibrium by adjusting $\log g$ (see, e.g., Koch \& McWilliam 2008 for an in-depth discussion of the relevant effects). In order to mend the discrepancies purely based on gravity, changes would require unrealistically low $\log g$ values as low as -0.2 dex for the coolest stars and gravities lower by 0.32 dex on average for the entire sample. This would settle the iron abundance at equally low values around $[\mathrm{Fe} / \mathrm{H}] \sim-2$ dex (see also Sect. 4; Table 4). If a false temperature scale was the cause for the disequilibrium, we would need to decrease the photometric $T_{\text {eff }}$ by $120 \mathrm{~K}$ on average, keeping all other parameters constant, to reinstall the ionization balance. The imbalance for the warmest stars alone is reconcilable with smaller changes in gravity or temperature that are compatible with the respective errors in these parameters. An unrealistically large depletion in the $\alpha$-elements in the atmospheres of the coolest stars by $>1$ dex could yield better agreement of the neutral and ionized species, but in the light of the regular $[\alpha / \mathrm{Fe}] \mathrm{ra}-$ tios found in the GC stars, we discard this explanation.

Similarly, we can exclude distance or mass errors as the source of the ionisiation imbalance: the stellar mass is well determined from the isochrones and would only have a minor influence on the gravity determinations if, for example, the targets were on the AGB. Likewise, the distance is well constrained from our CMD fit and all measurements agree to better than $5 \%$, 
which is too small to account for the required changes in $\log g$. Furthermore, the reddening from our CMD fit above is consistent with the value derived by Testa et al. (2001); moreover, the same value was obtained from the maps of Schlegel et al. (1998) maps with no indication of differential reddening.

None of the CMD features indicate any need for anomalous He abundances and we consider this an unlikely explanation for the observed imbalance. Thus, we conclude in keeping with Yong et al. (2003) that a combination of moderate stellar parameter changes is the most likely cause for the systematic differences in the $\mathrm{Fe} \mathrm{I}$ and $\mathrm{Fe}$ II abundances.

\subsubsection{NLTE and three-dimensional corrections}

Here we discuss whether the difference between $\varepsilon(\mathrm{Fe} I)$ and $\varepsilon(\mathrm{Fe}$ II $)$ could be a consequence of NLTE. To this end, we determined abundance corrections for $43 \mathrm{Fe}$ I and Fe II lines from Lind et al. (2012) and Bergemann et al. (2012). As a result, we find that the average neutral iron line abundance is affected at the 0.03 dex-level, while the ionized species suffer downward corrections of only 0.02 dex. Thus NLTE corrections could alleviate the ionization non-equilibrium at $0.05 \mathrm{dex}$. We caution, however, that these calculations were only possible for $\xi=2.0 \mathrm{~km} \mathrm{~s}^{-1}$ and $\log g=1.0$, owing to the limitations of the respective parameter grid in the sources above so that these corrections are slight extrapolations to the parameters of our stars.

Furthermore, we have undertaken an investigation to understand whether unaccounted three-dimensional (3D) atmosphere effects might explain the ionization imbalance resulting from our $1 \mathrm{D}$ analysis. While work on 3D model stellar atmospheres is not new (e.g., Nordlund 1982; Nordlund \& Dravins 1990), the work has been focused on the Sun and other dwarf stars. However, recent work by Magic et al. (2013a,b) has extended the grid of $3 \mathrm{D}$ stellar models to include metal-poor late-type stars, with $T_{\text {eff }}$ down to $4000 \mathrm{~K}$ and metallicities ranging from $[\mathrm{Fe} / \mathrm{H}]+0.5$ to -4.0 dex. Here, we make use of the grid of mean 3D stellar atmospheres (henceforth $\langle 3 \mathrm{D}\rangle$ ) of Magic et al. (2013b).

Magic et al. (2013a) and Bergemann et al. (2012) compared their $\langle 3 \mathrm{D}\rangle$ results with those from 1D atmospheres. Bergemann et al. (2012) used the Magic et al. (2013b) models to compute NLTE and $\langle 3 \mathrm{D}\rangle$ Fe I abundance corrections. The results indicate significant $\langle 3 \mathrm{D}\rangle$ corrections increasing for hotter, more metal-poor stars. In particular, larger corrections were seen for lines of lower excitation potential. In 1D LTE analyses, such effects would result in excitation temperatures determined from the spectra that are significantly higher than the actual effective temperatures. Notably, the $\langle 3 \mathrm{D}\rangle$ corrections were smallest for the Sun and relatively small for HD 122563, a cool, metal-poor RGB star $\left(T_{\text {eff }} / \log g /[\mathrm{Fe} / \mathrm{H}]\right.$ of $\left.4665 / 1.64 /-2.57\right)$.

We employed MOOG to compute the predicted EWs of our Fe I and Fe II lines using two Magic et al. (2013b) 〈3D $\rangle$ models: with $T_{\text {eff }} / \log g /[\mathrm{Fe} / \mathrm{H}] / \xi$ of $4500 \mathrm{~K}, 2.00,-2.00 \mathrm{dex}$, and $1.8 \mathrm{~km} \mathrm{~s}^{-1}$ and $4000 \mathrm{~K}, 1.50,-2.00 \mathrm{dex}$, and $1.8 \mathrm{~km} \mathrm{~s}^{-1}$. Unfortunately, the $\log g$ values of the Magic et al. (2013b) $\langle 3 \mathrm{D}\rangle$ grid only go down to 1.50 , somewhat higher than our GC RGB stars, whose gravities range from 0.44 to 1.38 .

In our calculations, we employed MOOG and the $\langle 3 \mathrm{D}\rangle$ atmospheres to compute predicted EWs for all Fe I and Fe II lines measured in our 1D analysis. We then followed our 1D abundance spectroscopic analysis method, using Kurucz models, to determine $T_{\text {eff }}, \xi$, and $[\mathrm{Fe} / \mathrm{H}]$ from the predicted $\langle 3 \mathrm{D}\rangle \mathrm{EWs}$.

For the warmer model, at $T_{\text {eff }} 4500, \log g 2.00$, it was necessary to reduce the temperature by $120 \mathrm{~K}$ in our 1D LTE analysis to ensure that the Fe I abundances were independent of line excitation potential; it was also necessary to increase our microturbulent velocity parameter, $\xi$, by $0.2 \mathrm{~km} \mathrm{~s}^{-1}$ so that the mean iron abundances were independent of EW. On average, we found that our 1D LTE analysis gave Fe I abundances lower than that input into the $\langle 3 \mathrm{D}\rangle \mathrm{EW}$ calculation by $0.09 \mathrm{dex}$, while the mean Fe II abundances were 0.03 dex lower for the $1 \mathrm{D}$ model calculations.

For our cooler $\langle 3 \mathrm{D}\rangle$ model, at $T_{\text {eff }} 4000$ and $\log g 1.50$, our $1 \mathrm{D}$ analysis required a temperature lower by $80 \mathrm{~K}$, but with the same microturbulent velocity as the $\langle 3 \mathrm{D}\rangle$ model at $1.8 \mathrm{~km} \mathrm{~s}^{-1}$. In this case, the resulting mean 1D LTE Fe I abundance was lower than the $\langle 3 \mathrm{D}\rangle$ input value by only $0.034 \mathrm{dex}$, while the mean Fe II abundance was lower by 0.014 dex. Thus, from our calculations we conclude that the $\langle 3 \mathrm{D}\rangle$ contribution to the ionization imbalance for $\mathrm{Fe}$ is in the range 0.02-0.06 dex.

While these $\langle 3 \mathrm{D}\rangle$ results cannot explain the ionization balance in the majority of our program stars, it is interesting that for the two stars with parameters closest to our calculations (S-290 and S-364) we measure 1D Fe I and Fe II abundances completely consistent with the small deviations expected from the $\langle 3 D\rangle$ models. Unfortunately, we cannot completely eliminate $\langle 3 \mathrm{D}\rangle$ effects as the source of our ionization imbalance, because we do not have $\langle 3 \mathrm{D}\rangle$ models with $\log g$ near 0.5 to 1.0 at $T_{\text {eff }}$ of $4000 \mathrm{~K}$ and $[\mathrm{Fe} / \mathrm{H}]=-2$. Still, our 1D LTE iron ionization imbalance is much larger than the computed $\langle 3 \mathrm{D}\rangle$ effect for relatively close stellar parameters; that is, if $\langle 3 D\rangle$ effects are responsible, there must be a dramatic increase in the effect for the coolest RGB stars. Furthermore, the small dispersion in the Fe I abundances of our sample indicates that the imbalance principally affects the ionized lines for the coolest stars.

\subsubsection{Model metallicity}

Initially, we assumed a mean metallicity of -1.7 dex from the slope of the RGB by Testa et al. (2001) as input for the model atmospheres. An independent estimate can be reached from two indicators.

First, we derived values for $[\mathrm{Fe} / \mathrm{H}]_{\mathrm{CaT}}$ from the near-infrared CaT lines at $8498 \AA, 8452 \AA$, and $8662 \AA$. These lines are a well-calibrated metallicity indicator for red giants in Galactic GCs (Armandroff \& Zinn 1988; Rutledge et al. 1997a,b; Carretta \& Gratton 1997; Starkenburg et al. 2010). The measurement of the CaT from high-resolution data, however, should be treated with caution: the dominant abundance information lies in the wings of the lines, while the cores are generally strongly saturated. These strong wings are easily affected by errors in the blaze function. Furthermore, any blends with weaker sky residuals, telluric lines, or neutral metal lines will affect their shapes; simplistic line profiles usually fail to fit reliably the CaT lines in very high-resolution spectra. Since their line cores form in the upper atmosphere and chromosphere, they are difficult to model reliably (e.g., Vernezza et al. 1976; McWilliam et al. 1995; Starkenburg et al. 2010). For a simple order-of-magnitude estimate, we numerically integrated the CaT lines over the band passes of Armandroff \& Zinn (1988). The thus measured EWs were then translated to metallicities on the scale of Carretta \& Gratton (1997) using the calibrations of Rutledge et al. (1997a,b) and, as an additional test, adopting the calibration of Starkenburg et al. (2010) which provides a more robust estimate for metalpoor stars.

As a second metallicity indicator, we integrated the MgIlines at $5167 \AA, 5173 \AA$ to calibrate $[\mathrm{Fe} / \mathrm{H}]$ on the 
scale of Carretta \& Gratton (1997) as $[\mathrm{Fe} / \mathrm{H}]_{\mathrm{MgI}}=-2.11+$ $1.76\left[\Sigma \mathrm{Mg}+0.079\left(V-V_{\mathrm{HB}}\right)\right]$. Here, $\Sigma \mathrm{Mg}=0.547\left(W_{5167}+\right.$ $\left.W_{5173}\right)$ denotes the $\mathrm{Mg}$ index as defined and calibrated in Walker et al. (2007). As a result, we find a mean CaT metallicity $[\mathrm{Fe} / \mathrm{H}]_{\mathrm{CaT}}$ of the seven giants of $-1.84 \pm 0.01 \mathrm{dex}$, and $-1.94 \pm 0.01$ dex on the scales of Carretta \& Gratton (1997) and Starkenburg et al. (2010), respectively. The latter provides a more reliable calibration at the metal-poor end. The mean $[\mathrm{Fe} / \mathrm{H}]_{\mathrm{Mg} \text { I }}$ of our stars is $-1.74 \pm 0.01 \mathrm{dex}$. The full range of 0.2 dex in these values illustrates that these methods should only be considered as order-of-magnitude guesses. While both the $\mathrm{CaT}$ and $\mathrm{Mg}$ I values are listed in Table 7, we point out that these values are only meant as initial metallicity estimates rather than reliable measures of NGC 5897's abundance scale.

To conclude, we used the abundance of the Fe I lines as input metallicity for the next iteration step as we iterated the above steps simultaneously in all parameters until convergence was reached.

\subsubsection{Differential analysis}

In order to test additional causes of the non-equilibrium, we also performed a differential abundance analysis, relative to a reference star of known stellar parameters. Here, we chose star 13414 in NGC 6397 (Koch \& McWilliam 2011), which lies close in parameter space to the present sample star $\mathrm{S}-9\left(T_{\mathrm{eff}}, \log g, \xi\right.$, $[\mathrm{Fe} / \mathrm{H}]=4124 \mathrm{~K}, 0.29,1.74,-2.14)$. Differential abundances were then obtained line by line using the line list of Koch \& McWilliam (2011) so that uncertainties in stellar parameters, but primarily the influence of atomic parameters and potential shortcomings in the model atmospheres, are reduced. The list has $\sim 75$, generally weaker, lines in common with the one used in the present study so that an independent confirmation of our parameters and results can be achieved. As before, we adopt the photometric temperature for this star as granted.

The main outcome from this exercise is that the microturbulence of S-9 needs to be lowered to $1.53 \mathrm{~km} \mathrm{~s}^{-1}$. The overall impact on the abundance results is small in that the differential iron abundance of S-9 decreases by $\sim 0.02$ dex, while the difference between neutral and ionized species remains, at $[\mathrm{Fe} \mathrm{I} / \mathrm{Fe} \mathrm{II}]=-0.26$.

\subsubsection{The variable star S-20}

Sandage \& Katem (1968) detected variability in star S-20 and classified it as a red semiregular variable. While Wehlau (1990) reports an overall amplitude of $0.3 \mathrm{mag}$ in $\mathrm{V}$, no period for the variability over the years 1956-1987 could be measured. We consulted additional data from the Catalina Surveys (CSS, CRTS; Drake et al. 2009), spanning the years 2006-2013, and find regular variations with an amplitude of $0.07 \mathrm{mag}$ in $\mathrm{V}$ and a period of 286 days, while individual events are seen as bright as $0.5 \mathrm{mag}$ above average. A detailed analysis of its lightcurve is, however, irrelevant to this work and in the following we assume that the stellar parameters of S-20 are sufficiently stable throughout our exposures, which cover a mere $30 \mathrm{~min}$, and that no systematic changes in the derived abundances were invoked. As the results in Sect. 5 imply, this star does not show any striking abundance anomalies or strong deviations from the cluster trends. We briefly discuss its abundance patterns within the overall GC environment in Sect. 5.7.

\section{Abundance errors}

To estimate the systematic errors in the chemical abundance ratios, we used the standard approach of computing nine new stellar atmospheres with altered stellar parameters - each $\left(T_{\text {eff }}\right.$, $\log g, \xi,[\mathrm{M} / \mathrm{H}],[\alpha / \mathrm{Fe}])$ was varied by its typical uncertainty (Sect. 3.1). To test the $\alpha$-enhancement, we re-ran the analysis using the solar-scaled opacity distributions, ODFNEW, translating to an uncertainty in the models' $[\alpha / \mathrm{Fe}]$ ratio of 0.4 dex. New element ratios were then determined and we list in Table 4 the differences in $[\mathrm{X} / \mathrm{Fe}]$ from those derived using the best-fit atmospheres as derived in Sect. 3.1. This procedure was performed for the stars S-255 and S-364, which bracket the full range in $T_{\text {eff }}$.

The total systematic $1 \sigma$ uncertainty, $\sigma_{\text {tot }}$, is obtained by summing all contributions in quadrature ${ }^{4}$, although this yields only a conservative upper limit; the realistic, underlying errors will be smaller because of the covariances of the atmosphere parameters, in particular, between temperature and gravity (e.g., McWilliam et al. 1995). As this exercise shows, the total systematic error on $[\mathrm{Fe} / \mathrm{H}]$ is 0.15 dex from the neutral and of the same order of magnitude for the ionized species. The $\alpha$-elements are uncertain to within 0.12 dex on average, but we refer to Table 4 to note differences between individual elements such as smaller errors for $\mathrm{Si}$ compared to larger ones on the $[\mathrm{Ti} / \mathrm{Fe}]$ ratios. Likewise, the heavier iron peak and neutron capture elements show systematic errors of 0.07-0.16 dex (quartile range), with the highest values for the iron-peak elements $\mathrm{V}$ and $\mathrm{Cr}$. This is in line with the low excitation potential of the lines of these elements, which leads to a strong dependence of their abundances on $T_{\text {eff }}$ (e.g., Ramírez \& Cohen (2003). Of all the elements, Ba shows the strongest trend with microturbulence due to its overall strong lines. None of our results is strongly affected by changes in the input metallicties, $[\mathrm{M} / \mathrm{H}]$, while changes in the $[\alpha / \mathrm{Fe}]$ ratio of the atmospheres have a greater impact only on the ionized species of the heavier elements at $Z \geq 56$.

To account for the random error due to uncertainties in the atomic parameters (mainly $\log g f$ ), the spectral noise, and potential insufficiencies in the atmosphere models themselves, we list in Table 5 the $1 \sigma$ line-to-line scatter and the number of lines used to derive each abundance ratio. For elements with many measured transitions, like $\mathrm{Fe}, \mathrm{Ca}, \mathrm{Sc}, \mathrm{Ti}, \mathrm{Cr}$, or $\mathrm{Ni}$, systematic uncertainties are the most dominant contribution, while elements with only a few well measured lines, the line-to-line scatter will be on par or even dominate the error budget. If only one line was detectable for a certain element, we conservatively adopted an uncertainty of 0.10 dex. For the following plots and statistics, all other elements were assigned a minimum random error of 0.05 dex (e.g., Ramírez \& Cohen 2003).

\section{Abundance results}

Table 5 lists the abundance ratios relative to Fe I, except for all ionized species and oxygen, which was measured from the [OI] lines. These are given relative to Fe II. This table also contains the average abundances and $1 \sigma$ dispersion of the entire RGB sample. Our results are also illustrated in the boxplot in Fig. 3 which shows the median and interquartile ranges for each element abundance ratio.

\footnotetext{
4 For this we adopted a quarter of the ODF error so as to mimic an uncertainty of the stars' $[\alpha / \mathrm{Fe}]$ ratio of 0.1 dex.
} 


\subsection{Iron}

Our study resulted in a mean iron abundance from the neutral species of $[\mathrm{Fe} / \mathrm{H}]=-2.04 \pm 0.01$ (stat.) \pm 0.15 (sys.), and $[\mathrm{Fe} / \mathrm{H}]=-1.92 \pm 0.03$ (stat.) \pm 0.15 (sys.) from the ionized lines. All previous studies have reported higher values for the GC metallicity: Testa et al. (2001) obtained $[\mathrm{Fe} / \mathrm{H}]=-1.7 \pm$ 0.16 from the slope of RGB; similar values are found on the scales of Zinn \& West (1984) and Rutledge et al. (1997), i.e., -1.68 and -1.73 dex, respectively. The most comprehensive low-resolution spectroscopic study of this GC to date is that of Geisler et al. (1995), who measured a mean metallicity of $-1.94 \pm 0.04$ from the CaT in 14 red giants. More recent spectroscopic data appear to converge on a more metal-poor mean compared to earlier photometric studies and we note here the value by Carretta et al. (2009a) of $-1.90 \pm 0.06$ and the scale of Kraft \& Ivans (2003) that reaches as low as -2.09 for NGC 5897.

Sobeck et al. (2011) also report on a downward correction of the $[\mathrm{Fe} / \mathrm{H}]$ value by $\sim 0.3$ dex of the metal-poor GC M15 compared to older spectroscopic studies. Furthermore, they found a discrepancy of up to 0.15 dex between the metallicities of their red HB and their RGB sample; reasons for the difference were sought in gravitational settling or the missing treatment of sphericity in the atmospheres, while uncertainties in the atmospheric parameters or atomic physics were excluded as possible causes. While we cannot test any systematic differences with evolutionary status in NGC 5897 with our pure RGB sample, downward corrections at the metal-poor end do not appear uncommon.

None of the studies mentioned above leaves any room for a significant iron or metallicity spread nor is this found in our data. The intrinsic $1 \sigma$ dispersion of NGC 5897 is $0.04 \pm 0.01$ dex and it is entirely explicable with the mass of this $\mathrm{GC}\left(M_{V}=-7.23\right)$, which allows for a minimum amount of stochastic, internal enrichment in its central potential well (e.g., Carretta et al. 2009a).

\subsection{Lithium}

We determined upper limits on $A(\mathrm{Li})$ from the region around the resonance line at $6707 \AA$, using the noise characteristics and expected extent of the absorption feature, which is usually not visible in spectra of cool, red giants. Our low $3 \sigma$ upper limits of $A(\mathrm{Li}) \lesssim 0.15$ are thus compatible with the full destruction of $\mathrm{Li}$ in the stellar interiors, as is also found in red giants of similar effective temperatures and luminosities in other GCs over a broad range of metallicities (e.g., Lind et al. 2009).

\subsection{Light elements: $\mathrm{O}, \mathrm{Na}, \mathrm{Al}, \mathrm{K}$}

The [O I] $6300 \AA$ A line can potentially suffer from contamination with telluric lines. To test this, we compared our spectra with spectra of the telluric standard star $\operatorname{HR} 7889$, taken under the same conditions as the GC targets. Thus, we confirm that the immediate region around the line in our stars is not affected by any telluric contamination.

Non-LTE corrections for sodium are scarcely available in the literature for our exact stellar parameter combinations, in particular the low gravities. Using the data by Lind et al. $(2011)^{5}$ for the warmer stars we confirmed that the corrections are on the order of -0.05 dex, averaged over the usually four Na-lines we used in the analysis. Figure 4 illustrates the well sampled

\footnotetext{
Taken from the authors' web-based database, www. inspect-stars. net.
}

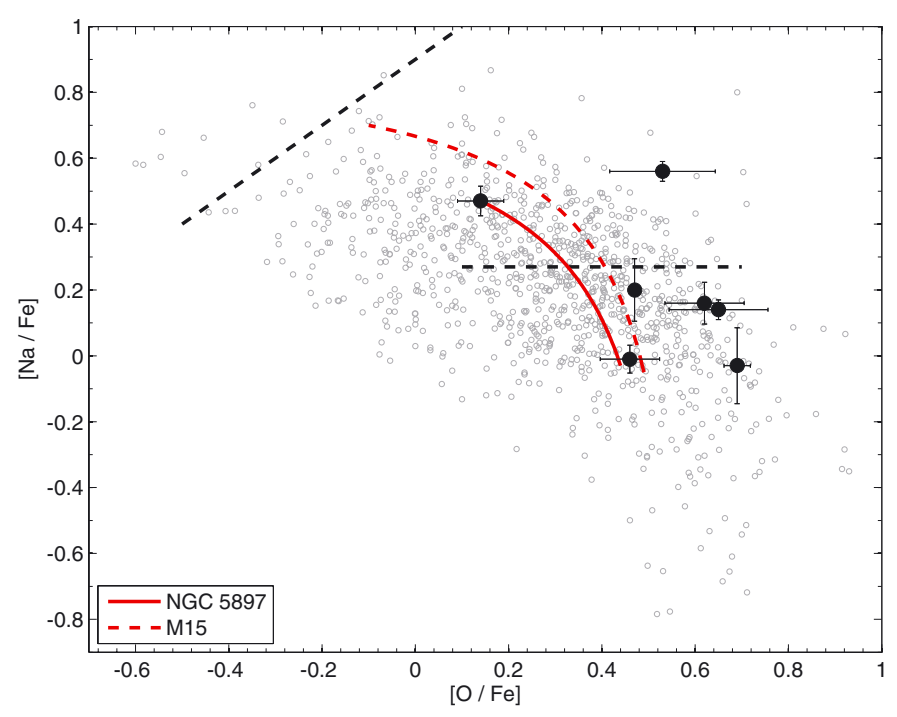

Fig. 4. Na-O anti-correlation in NGC 5897 (solid circles) on the data for Galactic GCs of Carretta et al. (2009b,c; gray points). The Na abundances have been corrected for NLTE, with typical corrections on the order of -0.05 dex. The dashed lines are empirical separations into primordial (lower part), intermediate (middle), and extreme (upper left corner) populations. Shown as a solid red line is the best-fit simple dilution model for NGC 5897, compared to the curve of the metal-poor GC M 15 from Carretta et al. (2009c; dashed red line).

$\mathrm{Na}-\mathrm{O}$ anti-correlation in NGC 5897, in comparison with the comprehensive sample for Galactic GCs compiled by Carretta et al. (2009b,c). Thus, NGC 5897 fully conforms with the bulk of GCs in that it shows chemical evidence of multiple stellar populations. Here, we indicate the empirical separations into the different generations of stars, established by Carretta et al. (2009c). Most of our sample can be dubbed primordial (P), as they fall below $[\mathrm{Na} / \mathrm{Fe}]_{\min }+0.3 \mathrm{dex}$, thus exhibiting the low $\mathrm{Na}-$ and high O-enhancements typical of stars that were only affected by the ejecta from early, massive SNe II without any contributions to the Na-budget. These coincide with the parameter space spanned by Galactic halo field stars. The remaining two giants already show indications of p-capture reactions that took place in the first-generation stars that ultimately enriched the ISM to form the second generation. Accordingly, these two giants of the intermediate (I) population are enhanced in $\mathrm{Na}$, with signs of lowered O levels. We note that the variable S-20 is characterized by the highest $\mathrm{Na} / \mathrm{Fe}$ ratio of our sample, while its $[\mathrm{O} / \mathrm{Fe}]$ ratio is not significantly depleted and also overlaps with the $\mathrm{P}$ generation.

Several studies have indicated that the ratio of first-to-second generation stars is approximately 30:70 (e.g., Carretta 2013). The fraction of 5:2 in our sample would rather imply the opposite trend, i.e., $70 \%$ of the stars belonging to the first generation. We caution, however, that this argument is hampered by our relatively sparse sample. The distinction into P and I components based on our seven giants may be inadequate as we may be missing first-generation stars even more depleted in Na. Thus, lowering $[\mathrm{Na} / \mathrm{Fe}]_{\min }$ would actually assign more of our targets to the second generation, thereby boosting the ratio to be more in line with most of the Galactic GCs studied to date.

The same holds when assessing the enrichment processes in this GC by computing simple dilution models (Carretta et al. 2009c). While we obtain a best fit to our few stars for $[\mathrm{Na} / \mathrm{Fe}]_{\max }=4.57$ and $[\mathrm{O} / \mathrm{Fe}]_{\max }=0.44$, a broader, true range in the $\mathrm{Na}-\mathrm{O}$ abundance space cannot be excluded at 

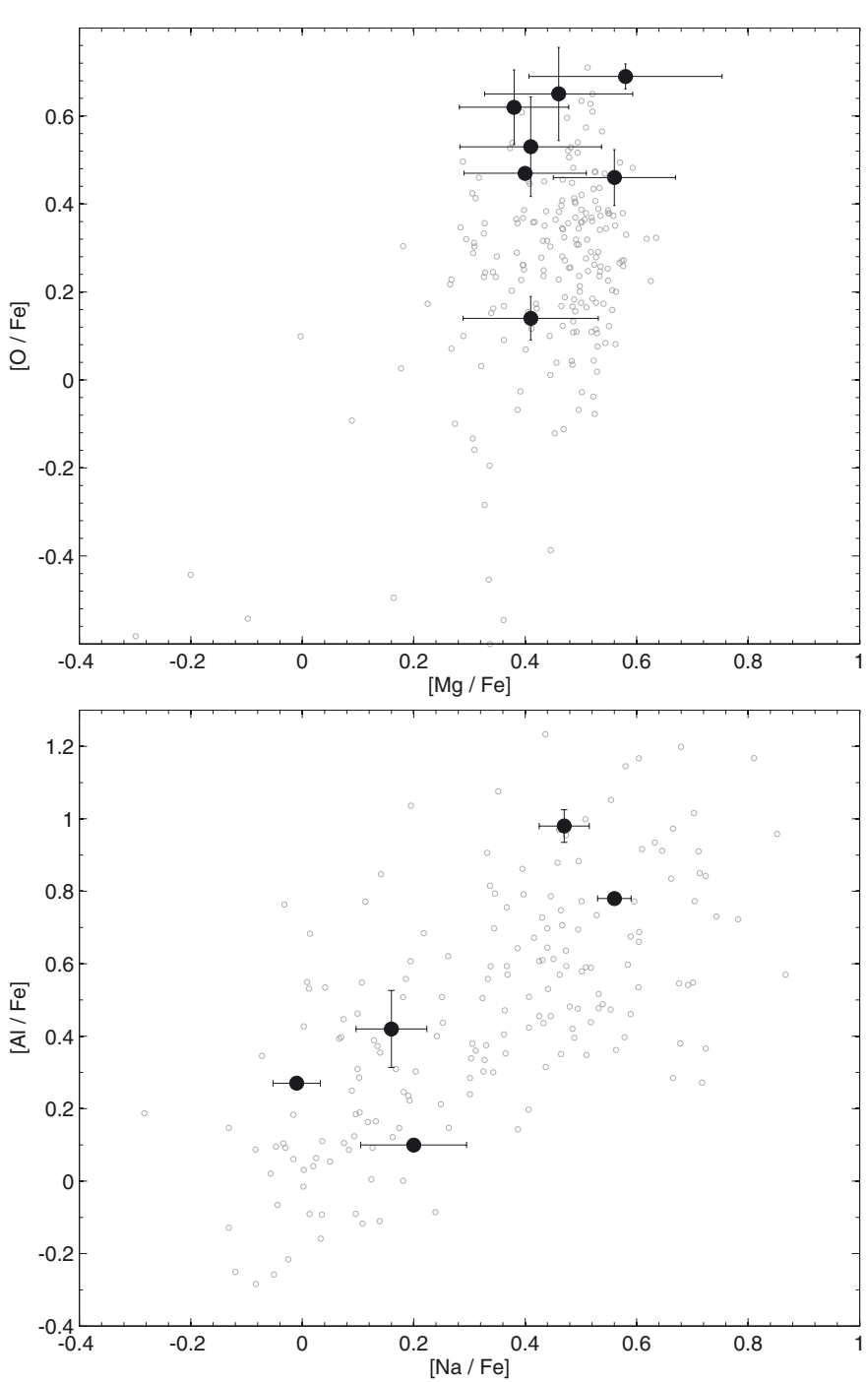

Fig. 5. Other p-capture element correlations using the same data as in Fig. 4.

present. Interestingly, NGC 5897 bears close resemblance to the Galactic halo GC M15, not only in terms of its metal-poor nature $\left([\mathrm{Fe} / \mathrm{H}]_{\mathrm{M} 15}=-2.6\right)$ and its location within the halo $\left(R_{\mathrm{GC}}=10.4 \mathrm{kpc}\right)$, but also when considering the dilution models in Na-O space in Fig. 4. Other, similarly metal-poor GCs covered by Carretta et al. (2009c) show much a broader range in the extrema of $\mathrm{Na}$ and/or $\mathrm{O}$, and/or steeper slopes in the $\mathrm{Na}-\mathrm{O}$ anticorrelation, such as NGC 6397. This suggests that a variety of factors besides metallicity is at play in shaping the details of the stellar generations seen in the GCs.

We note that $\mathrm{Al}$ measurements were only possible for five of the giants and that no clear $\mathrm{Mg}-\mathrm{Al}$ correlation is discernible. A common trend is maintained in that the star with the lowest $\mathrm{O}$ abundance also shows the highest enhancement in Al. There is other clear proof of the action of p-capture reactions among stars of the sample in NGC 5897: there is a clear Na-Al correlation (bottom panel of Fig. 5) in that the two giants with the highest $\mathrm{Na}$ abundance also have the highest $\mathrm{Al}$ abundance. This additional evidence strongly supports the presence of multiple stellar generations in this cluster. We note that any trend in $\mathrm{Mg}$ vs. $\mathrm{O}$ (top panel of Fig. 5) would be mainly driven by the O-poor star $\mathrm{S}-366$, which, however, still has a $[\mathrm{Mg} / \mathrm{Fe}]$ ratio compatible with the bulk of the GC stars. A correlation could be expected, since both elements are depleted in p-capture reactions.

Finally, no firm conclusions on trends of the potassium abundances can be reached from the strong resonance lines at $7664 \AA$, $7698 \AA$, as we do not sample the full extent of this GC's stellar populations (e.g., Mucciarelli et al. 2012) and those lines generally require a more detailed NLTE treatment (e.g., Takeda et al. 2009).

\section{4. $\alpha$-elements: $\mathrm{Mg}$, Si, Ca, Ti}

With regard to the elements primarily produced in Type II supernovae (SNe), NGC 5897 bears little surprises. We find a mean $[\alpha / \mathrm{Fe}]=\langle[\mathrm{Mg}, \mathrm{Ca}, \mathrm{Ti} \mathrm{I} / 3 \mathrm{Fe} \mathrm{I}]\rangle=0.34 \pm 0.01$ (stat.) \pm 0.12 (sys.), fully in line with the $\alpha$-enhancement found in Galactic halo GCs and field stars (e.g., Venn et al. 2004; Pritzl et al. 2005), rendering this object fully representative of its metallicity (e.g., Koch \& McWilliam 2011). NGC 5897 is also not unusual in that the intrinsic scatter in the $[\alpha / \mathrm{Fe}]$ ratios is well below 0.1 dex and thus explicable by the measurement errors.

Because of their production in the explosive SNe II events $(\mathrm{Ca}, \mathrm{Si}, \mathrm{Ti})$ and the hydrostatic burning of the progenitors $(\mathrm{Mg}, \mathrm{O})$, the $\alpha$-element abundances can be expected to trace each other, as is found in all major Galactic components (e.g., Fulbright et al. 2007). In this context, we measured $[\mathrm{Mg} / \mathrm{Ca}]$ and $[\mathrm{Ca} / \mathrm{Si}]$ ratios that are slightly elevated by 0.07 dex $(1 \sigma$ scatter of $0.07 \mathrm{dex}$ ), which compares to the values of around zero in Galactic disk and halo stars. However, the [Ca/Ti] ratio in our stars is notably higher, at $0.22 \mathrm{dex}$, and with a $1 \sigma$ scatter of 0.05 dex also remarkably homogeneous. As was argued, e.g., in Koch \& McWilliam (2010), this can be caused by an NLTE overionization of Ti I.

Star S-255 has $\alpha / \mathrm{Fe}$ ratios lower than the GC average, notably in $\mathrm{Si}$ and, to a lesser extent, in $\mathrm{Ca}$, but it does not stand out in the relative ratios of different $\alpha$-elements.

\subsection{Iron-peak and heavy elements: Sc, V, Mn, Cr, Co, Ni, $\mathrm{Cu}, \mathrm{Zn}$}

Like the $\alpha$-element distributions, none of the Fe-peak elements in NGC 5897 is outstanding. Because of the same production mechanism in SNe Ia, elements around the iron-peak are expected to follow $\mathrm{Fe}$. The $[\mathrm{Cr} / \mathrm{Fe}]$ and $[\mathrm{V} / \mathrm{Fe}]$ ratios in our stars are low (at $\sim-0.28$ to $-0.14 \mathrm{dex}$ ). We also find slightly subsolar values for $[\mathrm{Ni} / \mathrm{Fe}]$, whereas the $\mathrm{Co}-$ and $[\mathrm{Sc} / \mathrm{Fe}]$ ratios are slightly elevated and/or compatible with zero within the measurement errors.

At a mean of -0.43 dex and -0.70 dex, the $[\mathrm{Mn} / \mathrm{Fe}]$ and $[\mathrm{Cu} / \mathrm{Fe}]$ ratios are very low, yet typical of metal-poor halo field and GC stars. Overall, the Fe-peak elements are compatible with other GCs at similar metallicities and fall within the halo trends of, e.g., Cayrel et al. (2004) and Ishigaki et al. (2013); see also the discussions in Koch \& Côté (2010).

Nissen \& Schuster (2011) reported on systematic differences in the abundance distribution of halo stars between $-1.6<$ $[\mathrm{Fe} / \mathrm{H}]<-0.4$ in the solar neighborhood, which are most pronounced in $\mathrm{Na}, \mathrm{Cu}$, and $\mathrm{Zn}$. This was extended into the metalpoor regime $([\mathrm{Fe} / \mathrm{H}]>-3.3)$ by Ishigaki et al. (2013), confirming the inner/outer, or, in-situ/accreted halo dichotomy in their $\mathrm{Na} / \mathrm{Fe}$ and $\mathrm{Zn} / \mathrm{Fe}$ ratios with the strongest signatures again occurring above -1.5 dex. At the low metallicity of NGC 5897 we cannot unambiguously make such a distinction and, in a one-toone comparison with the halo samples, different elements would 


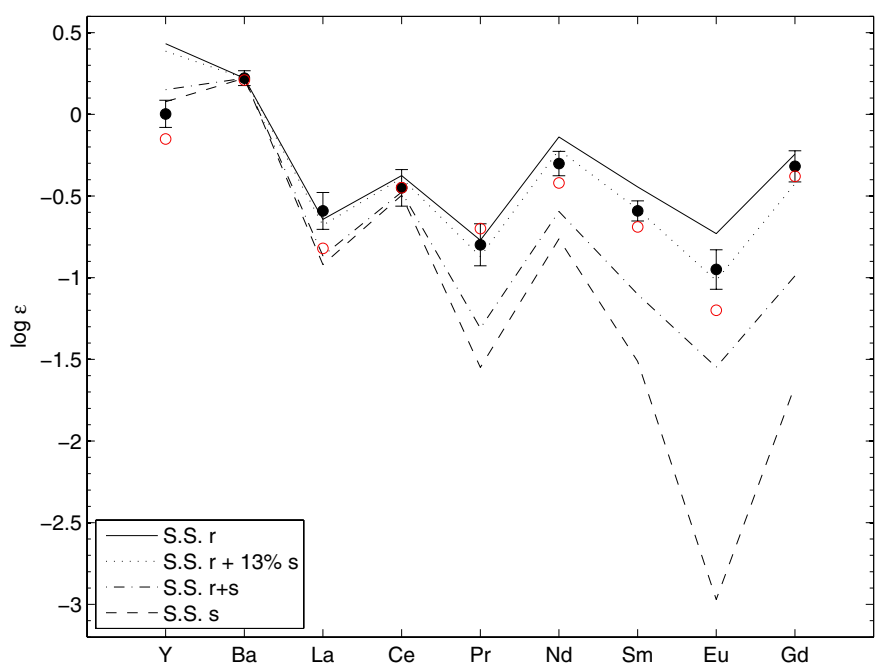

Fig. 6. Mean heavy element $(Z>38)$ distribution of the NGC 5897 stars. The different lines are the solar-scaled (S.S.) abundances from Simmerer et al. (2004), normalized to Ba. We also indicate the best fit to our measurements that is obtained by admixing $13 \%$ of s-process material. The red open circles show the abundances for star S-138, which is low in Eu.

yield different associations with either halo component (e.g., a low $[\mathrm{Cu} / \mathrm{Fe}]$ more in line with the low- $\alpha$, accreted population while our elevated $[\mathrm{Zn} / \mathrm{Fe}]$ ratio is reminiscent of the high- $\alpha$ halo stars that likely formed in-situ).

\subsection{Neutron-capture elements: Y, Ba, La, Ce, Pr, Nd, Sm, $\mathrm{Eu}, \mathrm{Gd}$}

We could reliably measure abundance ratios of nine chemical elements heavier than $Z>38$. As often stated for other elements above, their mean values are also normal and compatible with those of other GCs and field halo stars of the same metallicity (Pritzl et al. 2005; Tolstoy et al. 2009; Kacharov et al. 2013). In this regard, we measure an $[\mathrm{r} / \mathrm{s}]$ ratio, represented by $[\mathrm{Eu} / \mathrm{Ba}]$, of $0.48 \mathrm{dex}$, which lies close to the solar-scaled r-process value (e.g., Simmerer et al. 2004), as can be expected, since at these low metallicities AGB contributions were few and the bulk of the heavy elements was produced in the r-process (e.g, Truran $1981)^{6}$.

We note that none of our targets is exceptionally Ba-rich; the fact that the intermediate-population, Na-rich stars also have normal $[\mathrm{Ba} / \mathrm{Fe}]$ ratios shows that they have not been enriched by great amounts of low-mass AGB ejecta that could have formed late in the central regions of the GC (cf. Kacharov et al. 2013).

As Fig. 6 indicates, the mean heavy element abundances in NGC 5897 lie close to the solar-scaled r-process curve (taken from Simmerer et al. 2004). An exception is yttrium, which lies closer to the solar s-process curve; Y, like its neighbour $\mathrm{Sr}$, is associated with an additional, unknown, nucleosynthetic process, first identified by McWilliam (1998), and now often referred to as lighter element primary process (LEPP; e.g. Travaglio et al. 2004). The best fit to the production of these heavy elements can be obtained for a scaled-solar pure r-process with an admixture of about $13 \%$ of s-process material (see also Kacharov et al. 2013). This also accounts well for $\mathrm{Zn}$, which is thought to have contributions from massive $\mathrm{SNe}$ II at very low metallicities as

\footnotetext{
6 The s-process, however, might have been active in the Galaxy as early as $[\mathrm{Fe} / \mathrm{H}]>-2.6$ (Simmerer et al. 2004).
}

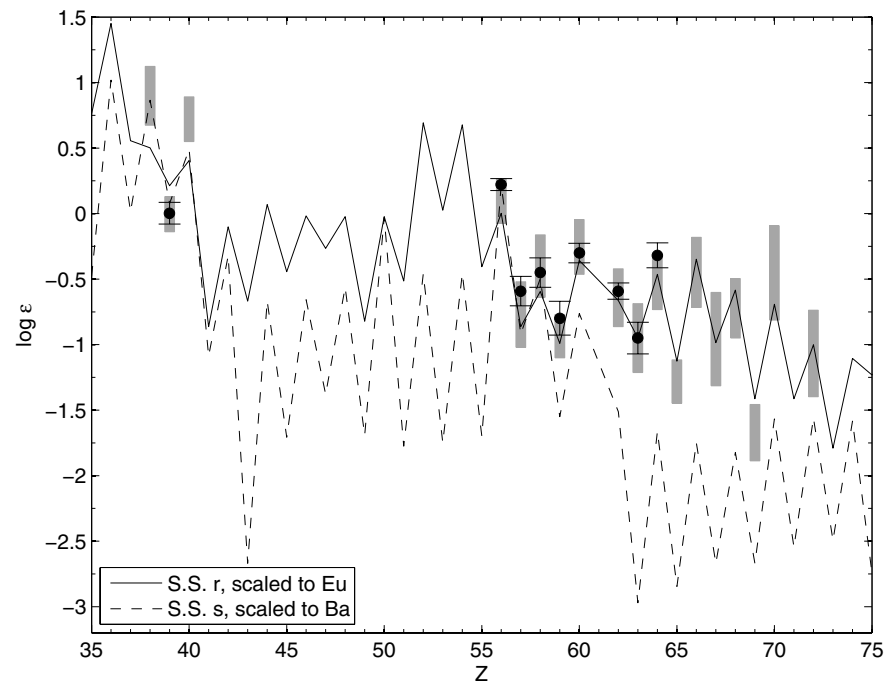

Fig. 7. Comparison of NGC 5897 (solid circles) with the heavy element abundances of M15 (Sobeck et al. 2011), where the gray shaded areas frame their $1 \sigma$ ranges. In contrast to Fig. 6 , the solar-scaled curve for the r-process is shown relative to $\mathrm{Eu}$, while the s-process curve is still normalized to $\mathrm{Ba}$.

well as nucleosynthesis in the weak s-process in massive stars, while low-mass AGB production appears to be negligible in this regime of NGC 5897 (e.g., Timmes et al. 1995; Ishigaki et al. 2013). Other GCs show a clear-cut r-process dominance that remains even until higher metallicities (e.g., Pal 3, M15, M75; Koch et al. 2009; Sneden et al. 2000; Kacharov et al. 2013) and has been linked to the loose central concentration of those objects in the outer halo.

The abundance mix we find in NGC 5897 at its low metallicity is fully consistent with the notion that only a few AGB stars have contributed to the enrichment of the material out of which the GC formed at early times. To emphasize this, we overplot in Fig. 7 the mean distribution of heavy elements in M15 by Sobeck et al. (2011), who determined abundance ratios of 22 neutroncapture elements in this metal-poor $([\mathrm{Fe} / \mathrm{H}] \sim-2.6)$ halo GC. The similarity of these elements measured in the two studies is obvious and clearly compatible with the negligible contributions by s-process nucleosynthesis.

\subsection{Notes on individual stars}

Here, we comment on those stars among the otherwise regular sample that show one or the other noteworthy abundance ratios.

S-255: this is the coolest and most metal-rich star of our sample. Some of its $[\alpha / \mathrm{Fe}]$ abundance ratios are significantly lower than the cluster mean (by $\sim 0.15 \mathrm{dex}$ ), in particular $\mathrm{Si}$ and $\mathrm{Ca}$. Consequently, depletions are also found for $\mathrm{K}$ and $\mathrm{Sc}$ and some of the heavier elements (Y, La).

S-20/V-5: the variable star candidate shows the highest $\mathrm{Na}$ abundance in our sample, albeit at a regular $\mathrm{O} / \mathrm{Fe}$ ratio. An association with the intermediate GC population is thus not unambiguous.

S-366: this star is the best contender for a true secondgeneration star, given its very low $\mathrm{O}$ - and very high $\mathrm{Na}$ abundances. None of its other element ratios are outstanding in any regard.

S-138: with the highest $\mathrm{O}$ and a low $\mathrm{Na}$ abundance, this star is a good representative of the primordial GC population. What distinguishes this object, however, are $\mathrm{Eu}$ and $\mathrm{La}$ abundances 


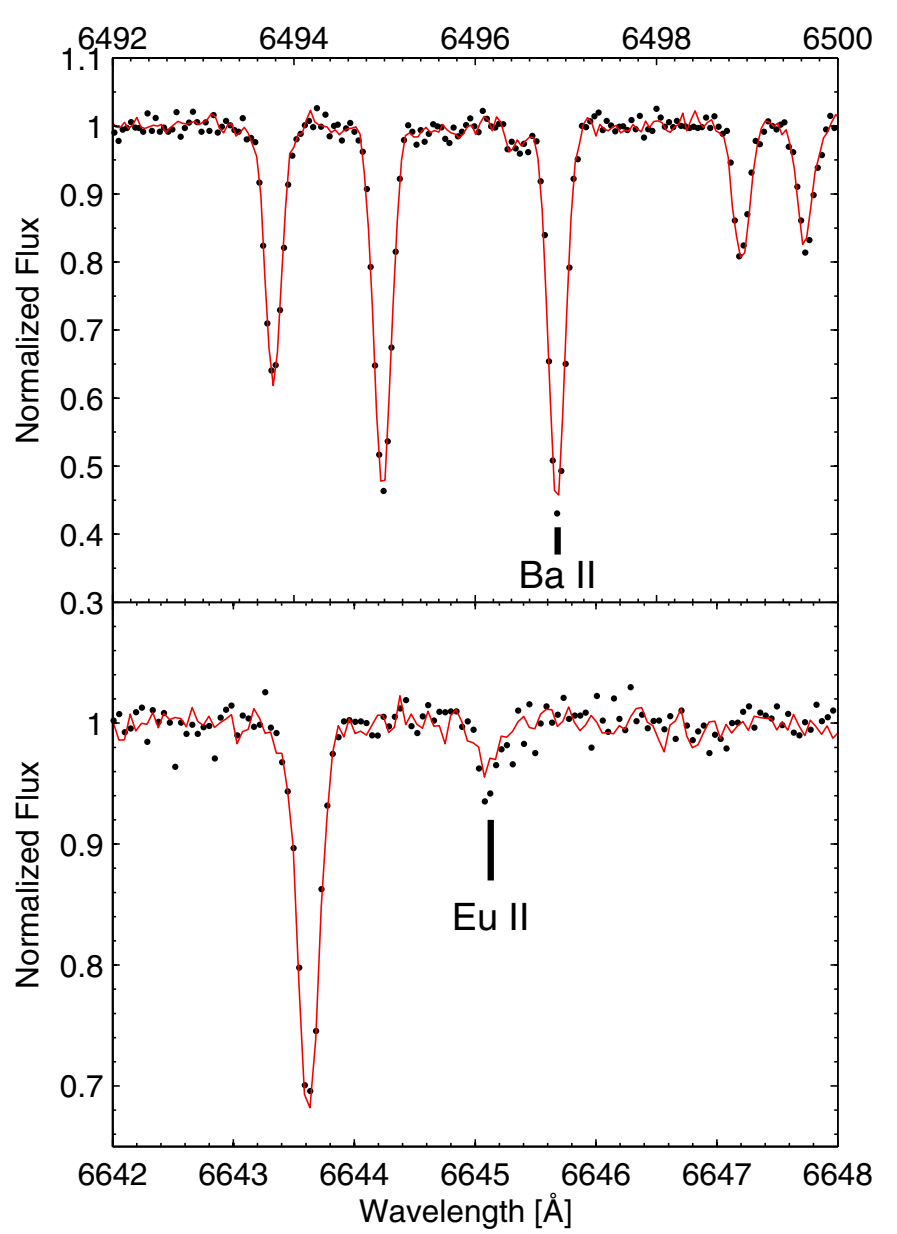

Fig. 8. Comparison of the Ba II $6496.9 \AA$ line (top panel) and Eu II $6645 \AA$ line (bottom panel) in two stars with similar stellar parameters: S-290 (black dots) and the primordial star S-138 (solid red line), which shows low $\mathrm{Na}, \mathrm{La}, \mathrm{Eu}$, and $\mathrm{Nd}$ abundances.

that are significantly lower than in the remainder of the GC stars analyzed here (see Figs. 6, 8), while $\mathrm{Ba}$ is higher than in the remainder of our sample. This is highlighted in Fig. 8, where we compare two absorption features for $\mathrm{Ba}$ and Eu used in our abundance analysis for the Eu-poorer S-138 and a typical cluster representative, S-2907. Eu can be depleted very easily because of its large neutron-capture cross section, similar to La, which contains a larger $\mathrm{r}$-process fraction relative to $\mathrm{Ba}$.

Accordingly, S-138 stands out in that it has a $[\mathrm{Ba} / \mathrm{Eu}]$ that is higher by 0.28 dex than the other six red giants. On the other hand, its [La/Y] (thus a [hs/ls]) ratio of 0.41 is consistent with the GC's mean value, indicating that this star was not polluted by an unusual mass range of progenitors, but rather the same sources (e.g., low-intermediate mass, metal-poor AGB stars; Cristallo et al. 2009) produced the observed abundance patterns.

\section{Summary}

Our abundance analysis of a large number of chemical tracers in the metal-poor inner halo cluster NGC 5897 identified this GC as fully representative of the bulk of such objects in the Milky Way.

\footnotetext{
7 A similar, obvious depletion is seen for the stronger Eu II line at $4129 \AA$, which is, however, hampered by difficulties in the continuum setting due to blending, and thus was not used in our abundance analysis.
}

This includes the enhancement in the $\alpha$-elements to $\sim 0.3 \mathrm{dex}$, approximately solar Fe-peak elements, and n-capture elements that were predominantly produced by the r-process. The latter notion is consistent with only negligible AGB contributions to the chemical inventory in the metal-poor (read: early-time) regime. The presence of a pronounced $\mathrm{Na}-\mathrm{O}$ anti-correlation renders this object, by definition, a globular cluster (not that there was ever a doubt). Unfortunately, our limited sample size did not allow us to trace the full extent of this relation; in particular, we are lacking information about the spatial variations of the first vs. second generations, but the severe crowding prohibited exposures of the central stars. All other elements, except for $\mathrm{O}, \mathrm{Na}$, and $\mathrm{Al}$ show little star-to-star scatter, mostly explicable by the measurement uncertainties or in part driven by peculiar patterns in individual stars. Several features, such as the shape of the $\mathrm{Na}-\mathrm{O}$ correlation and the preponderance of the r-process, are reminiscent of the metal-poor $([\mathrm{Fe} / \mathrm{H}] \sim-2.6 \mathrm{dex})$ halo $\mathrm{GC}$ M15.

Our work has revealed NGC 5897 to be more metal poor than implied by older CMD and low-resolution metallicity studies. We suggest that this lower metallicity can explain the remarkably longer periods of the RR Lyr found in this GC (but see Hansen et al. 2011 for the Period-metallicity relation of extremely metal-poor stars at $[\mathrm{Fe} / \mathrm{H}] \sim-3)$. The error-weighted mean metallicity of the RR Lyr of Clement \& Rowe (2001) is $[\mathrm{Fe} / \mathrm{H}]=-2.2 \pm 0.2$ on the scale of Zinn \& West (1984), where we adopted the relations between period, phase, and metallicity of Jurcsik (1995); see also Haschke et al. (2012). While the errors for individual stars are tremendous, the mean value of the RR Lyr is thus in line with the metal-poor nature of this GC found from our red giants.

Acknowledgements. A.K. acknowledges the Deutsche Forschungsgemeinschaft for funding from Emmy-Noether grant Ko 4161/1. We thank the anonymous referee for a helpful and constructive report. This research made use of atomic data from the INSPECT database, version 1.0 (www . inspect-stars.net). The CSS survey is funded by the National Aeronautics and Space Administration under Grant No. NNG05GF22G issued through the Science Mission Directorate Near-Earth Objects Observations Program. The CRTS survey is supported by the US National Science Foundation under grants AST-0909182 and AST-1313422.

\section{References}

Armandroff, T. E., \& Zinn, R. 1988, AJ, 96, 92

Asplund, M., Grevesse, N., Sauval, A. J., \& Scott, P. 2009, ARA\&A, 47, 481

Bergemann, M., Lind, K., Collet, R., Magic, Z., \& Asplund, M. 2012, MNRAS, 427, 27

Burris, D. L., Pilachowski, C. A., Armandroff, T. E., et al. 2000, ApJ, 544, 302

Busso, M., Gallino, R., Lambert, D. L., Travaglio, C., \& Smith, V. V. 2001, ApJ, 557,802

Carretta, E. 2013, A\&A, 557, A128

Carretta, E., \& Gratton, R. 1997, A\&AS, 121, 95

Carretta, E., Bragaglia, A., Gratton, R., D’Orazi, V., \& Lucatello, S. 2009a, A\&A, 508, 695

Carretta, E., Bragaglia, A., Gratton, R., \& Lucatello, S. 2009b, A\&A, 505, 139

Carretta, E., Bragaglia, A., Gratton, R. G., et al. 2009c, A\&A, 505, 117

Carretta, E., Bragaglia, A., Gratton, R. G., et al. 2010, A\&A, 516, A55

Clement, C. M., \& Rowe, J. F. 2001, AJ, 122, 1464

Clement, C. M., Muzzin, A., Dufton, Q., et al. 2001, AJ, 122, 2587

Cristallo, S., Straniero, O., Gallino, R., et al. 2009, ApJ, 696, 797

Cutri, R. M. 2003, Explanatory Supplement to the 2MASS All-Sky Data Release, http://www.ipac/caltech.edu/2mass/releases/allsky/ doc/explsup.html

Drake, A. J., Djorgovski, S. G., Mahabal, A., et al. 2009, ApJ, 696, 870

D'Ercole, A., Vesperini, E., D'Antona, F., McMillan, S. L. W., \& Recchi, S. 2008, MNRAS, 391, 825

Decressin, T., Meynet, G., Charbonnel, C., Prantzos, N., \& Ekström, S. 2007, A\&A, 464, 1029

Dotter, A., Chaboyer, B., Jevremović, D., et al. 2008, ApJS, 178, 89 
Fabbian, D., Nissen, P. E., Asplund, M., Pettini, M., \& Akerman, C. 2009, A\&A, 500,1143

Fulbright, J. P., McWilliam, A., \& Rich, R. M. 2007, ApJ, 661, 1152

Fusi Pecci, F., Ferraro, F. R., Bellazzini, M., et al. 1993, AJ, 105, 1145

Geisler, D., Minniti, D., \& Claria, J. J. 1992, AJ, 104, 627

Geisler, D., Piatti, A. E., Claria, J. J., \& Minniti, D. 1995, AJ, 109, 605

Gratton, R. G., Carretta, E., Bragaglia, A., Lucatello, S., \& D’Orazi, V. 2010, A\&A, 517, A81

Gratton, R. G., Carretta, E., \& Bragaglia, A. 2012, A\&ARv, 20, 50

Hansen, C. J., Nordström, B., Bonifacio, P., et al. 2011, A\&A, 527, A65

Haschke, R., Grebel, E. K., Duffau, S., \& Jin, S. 2012, AJ, 143, 48

Ishigaki, M. N., Aoki, W., \& Chiba, M. 2013, ApJ, 771, 67

Jurcsik, J. 1995, Acta Astron., 45, 653

Kacharov, N., Koch, A., \& McWilliam, A. 2013, A\&A, 554, A81

Kelson, D. D. 2003, PASP, 115, 688

Kelson, D. D., Illingworth, G. D., van Dokkum, P. G., \& Franx, M. 2000, ApJ, 531,159

Koch, A., \& Côté, P. 2010, A\&A, 517, A59

Koch, A., \& McWilliam, A. 2008, AJ, 135, 1551

Koch, A., \& McWilliam, A. 2011, AJ, 142, 63

Koch, A., Côté, P., \& McWilliam, A. 2009, A\&A, 506, 729

Kraft, R. P., \& Ivans, I. I. 2003, PASP, 115, 143

Lawler, J. E., Guzman, A., Wood, M. P., Sneden, C., \& Cowan, J. J. 2013, ApJS, 205,11

Lind, K., Primas, F., Charbonnel, C., Grundahl, F., \& Asplund, M. 2009, A\&A, 503,545

Lind, K., Asplund, M., Barklem, P. S., \& Belyaev, A. K. 2011, A\&A, 528, A103

Lind, K., Bergemann, M., \& Asplund, M. 2012, MNRAS, 427, 50

Magic, Z., Collet, R., Hayek, W., \& Asplund, M. 2013a, A\&A, 560, A8

Magic, Z., Collet, R., Asplund, M., et al. 2013b, A\&A, 557, A26

McWilliam, A. 1998, AJ, 115, 1640

McWilliam, A., Preston, G. W., Sneden, C., \& Searle, L. 1995, AJ, 109, 2757

Mucciarelli, A., Bellazzini, M., Ibata, R., et al. 2012, MNRAS, 426, 2889

Nissen, P. E., \& Schuster, W. J. 2011, A\&A, 530, A15

Nordlund, Å. 1982, A\&A, 107, 1

Nordlund, A., \& Dravins, D. 1990, A\&A, 228, 155
Pietrinferni, A., Cassisi, S., Salaris, M., \& Castelli, F. 2004, ApJ, 612, 168 Piotto, G. 2009, IAU Symp., 258, 233

Pritzl, B. J., Venn, K. A., \& Irwin, M. 2005, AJ, 130, 2140

Raiteri, C. M., Gallino, R., Busso, M., Neuberger, D., \& Kaeppeler, F. 1993, ApJ, 419,207

Ramírez, I., \& Meléndez, J. 2005, ApJ, 626, 465

Ruland, F., Biehl, D., Holweger, H., Griffin, R., \& Griffin, R. 1980, A\&A, 92, 70

Rutledge, G. A., Hesser, J. E., Stetson, P. B., et al. 1997a, PASP, 109, 883

Rutledge, G. A., Hesser, J. E., \& Stetson, P. B. 1997b, PASP, 109, 907

Sadakane, K., Arimoto, N., Ikuta, C., et al. 2004, PASJ, 56, 1041

Sandage, A., \& Katem, B. 1968, ApJ, 153, 569

Schlegel, D. J., Finkbeiner, D. P., \& Davis, M. 1998, ApJ, 500, 525

Shetrone, M., Venn, K. A., Tolstoy, E., et al. 2003, AJ, 125, 684

Simmerer, J., Sneden, C., Cowan, J. J., et al. 2004, ApJ, 617, 1091

Sneden, C. A. 1973, Ph.D. Thesis, The University of Texas at Austin, USA

Sneden, C., Pilachowski, C. A., \& Kraft, R. P. 2000, AJ, 120, 1351

Sobeck, J. S., Kraft, R. P., Sneden, C., et al. 2011, AJ, 141, 175

Starkenburg, E., Hill, V., Tolstoy, E., et al. 2010, A\&A, 513, A34

Stetson, P. B., Bruntt, H., \& Grundahl, F. 2003, PASP, 115, 413

Takeda, Y., Kaneko, H., Matsumoto, N., et al. 2009, PASJ, 61, 563

Testa, V., Corsi, C. E., Andreuzzi, G., et al. 2001, AJ, 121, 916

Timmes, F. X., Woosley, S. E., \& Weaver, T. A. 1995, ApJS, 98, 617

Tolstoy, E., Hill, V., \& Tosi, M. 2009, ARA\&A, 47, 371

Travaglio, C., Gallino, R., Arnone, E., et al. 2004, ApJ, 601, 864

Truran, J. W. 1981, A\&A, 97, 391

Venn, K. A., Irwin, M., Shetrone, M. D., et al. 2004, AJ, 128, 1177

Vernazza, J. E., Avrett, E. H., \& Loeser, R. 1976, ApJS, 30, 1

Wehlau, A. 1990, AJ, 99, 250

Winkler, H. 1997, MNRAS, 287, 481

Wood, M. P., Lawler, J. E., Sneden, C., \& Cowan, J. J. 2013, ApJS, 208, 27

Yong, D., Grundahl, F., Lambert, D. L., Nissen, P. E., \& Shetrone, M. D. 2003, A\&A, 402, 985

Yong, D., Grundahl, F., Nissen, P. E., Jensen, H. R., \& Lambert, D. L. 2005, A\&A, 438, 875

Zinn, R., \& West, M. J. 1984, ApJS, 55, 45 
A. Koch and A. McWilliam: Chemical abundances in NGC 5897

Table 5. Abundance results for individual stars and the entire sample.

\begin{tabular}{|c|c|c|c|c|c|c|c|c|c|c|c|c|}
\hline \multirow{2}{*}{ Element $^{a}$} & \multicolumn{3}{|c|}{ S-255 } & \multicolumn{3}{|c|}{ S-20 } & \multicolumn{3}{|c|}{ S-9 } & \multicolumn{3}{|c|}{ S-366 } \\
\hline & {$[\mathrm{X} / \mathrm{Fe}]$} & $\sigma$ & $N$ & {$[\mathrm{X} / \mathrm{Fe}]$} & $\sigma$ & $N$ & {$[\mathrm{X} / \mathrm{Fe}]$} & $\sigma$ & $N$ & {$[\mathrm{X} / \mathrm{Fe}]$} & $\sigma$ & $N$ \\
\hline $\mathrm{Fe}_{\mathrm{CaT}}^{b}$ & -1.95 & $\ldots$ & $\cdots$ & -1.88 & $\ldots$ & $\ldots$ & -1.88 & $\cdots$ & $\ldots$ & -1.95 & $\cdots$ & .. \\
\hline $\mathrm{Fe}_{\mathrm{MgI}}^{c}$ & -1.73 & $\ldots$ & $\ldots$ & -1.76 & $\ldots$ & $\ldots$ & -1.70 & $\ldots$ & $\ldots$ & -1.77 & $\ldots$ & $\ldots$ \\
\hline Fe I & -1.99 & 0.23 & 133 & -2.05 & 0.21 & 136 & -2.05 & 0.14 & 129 & -2.02 & 0.20 & 131 \\
\hline Fe II & -1.77 & 0.11 & 13 & -1.89 & 0.11 & 12 & -1.85 & 0.12 & 13 & -1.92 & 0.20 & 13 \\
\hline$A(\mathrm{Li})$ & $<-0.66$ & & 1 & $<-0.62$ & $\ldots$ & 1 & $<-0.50$ & & 1 & $<-0.19$ & & 1 \\
\hline O I & 0.47 & 0.02 & 3 & 0.53 & 0.16 & 2 & 0.46 & 0.09 & 2 & 0.14 & 0.07 & 2 \\
\hline Na $\mathrm{I}^{\text {NLTE }}$ & 0.20 & 0.19 & 4 & 0.56 & 0.06 & 4 & -0.01 & 0.06 & 2 & 0.47 & 0.09 & 4 \\
\hline Mg I & 0.40 & 0.19 & 3 & 0.41 & 0.22 & 3 & 0.56 & 0.19 & 3 & 0.41 & 0.21 & 3 \\
\hline Al I & 0.10 & $\ldots$ & 1 & 0.78 & 0.03 & 4 & 0.27 & & 1 & 0.98 & 0.09 & 4 \\
\hline Si I & -0.04 & 0.29 & 4 & 0.25 & 0.26 & 4 & 0.24 & 0.29 & 4 & 0.20 & 0.30 & 4 \\
\hline KI & 0.24 & 0.01 & 2 & 0.39 & 0.03 & 2 & 0.61 & 0.06 & 2 & 0.64 & 0.07 & 2 \\
\hline $\mathrm{Ca} \mathrm{I}$ & 0.32 & 0.18 & 17 & 0.40 & 0.19 & 17 & 0.39 & 0.11 & 17 & 0.42 & 0.08 & 17 \\
\hline Sc II & -0.05 & 0.07 & 7 & 0.08 & 0.09 & 7 & 0.06 & 0.06 & 7 & 0.15 & 0.09 & 7 \\
\hline Ti I & 0.15 & 0.23 & 18 & 0.11 & 0.22 & 18 & 0.13 & 0.20 & 18 & 0.15 & 0.19 & 18 \\
\hline Ti II & 0.17 & 0.11 & 6 & 0.25 & 0.14 & 6 & 0.37 & 0.23 & 6 & 0.31 & 0.19 & 6 \\
\hline V I & -0.16 & 0.16 & 6 & -0.12 & 0.14 & 6 & -0.16 & 0.15 & 6 & -0.17 & 0.10 & 3 \\
\hline $\mathrm{Cr} \mathrm{I}$ & -0.25 & 0.09 & 7 & -0.19 & 0.11 & 7 & -0.12 & 0.13 & 7 & -0.17 & 0.08 & 7 \\
\hline Mn I & -0.39 & 0.13 & 6 & -0.41 & 0.15 & 6 & -0.41 & 0.10 & 6 & -0.40 & 0.05 & 6 \\
\hline CoI & 0.06 & 0.11 & 4 & 0.13 & 0.10 & 4 & 0.06 & 0.13 & 4 & -0.01 & 0.14 & 4 \\
\hline $\mathrm{Ni}$ I & -0.09 & 0.22 & 26 & -0.05 & 0.24 & 26 & -0.04 & 0.24 & 26 & -0.08 & 0.22 & 26 \\
\hline $\mathrm{Cu} \mathrm{I}$ & -0.71 & 0.13 & 3 & -0.61 & 0.05 & 3 & -0.71 & 0.11 & 2 & -0.62 & 0.04 & 2 \\
\hline $\mathrm{ZnI}$ & 0.09 & 0.11 & 2 & 0.10 & 0.06 & 2 & 0.12 & 0.19 & 2 & 0.08 & 0.08 & 2 \\
\hline Y II & -0.46 & 0.20 & 5 & -0.29 & 0.14 & 5 & -0.35 & 0.13 & 5 & -0.19 & 0.10 & 5 \\
\hline Ba II & -0.16 & 0.03 & 3 & -0.12 & 0.02 & 3 & -0.03 & 0.11 & 3 & 0.01 & 0.10 & 3 \\
\hline La II & 0.02 & 0.13 & 5 & 0.19 & 0.08 & 5 & 0.11 & 0.09 & 5 & 0.28 & 0.08 & 5 \\
\hline Ce II & -0.22 & $\ldots$ & 1 & -0.28 & $\ldots$ & 1 & -0.22 & $\ldots$ & 1 & -0.20 & $\ldots$ & 1 \\
\hline PrII & 0.15 & $\ldots$ & 1 & 0.43 & $\ldots$ & 1 & 0.16 & $\ldots$ & 1 & 0.57 & $\ldots$ & 1 \\
\hline Nd II & 0.08 & $\ldots$ & 1 & 0.22 & $\ldots$ & 1 & 0.09 & $\ldots$ & 1 & 0.16 & & 1 \\
\hline Sm II & 0.22 & 0.07 & 6 & 0.36 & 0.07 & 5 & 0.26 & 0.05 & 5 & 0.34 & 0.08 & 6 \\
\hline Eu II & 0.40 & 0.05 & 2 & 0.40 & 0.02 & 2 & 0.40 & 0.07 & 2 & 0.56 & 0.06 & 2 \\
\hline Gd II & 0.45 & $\ldots$ & 1 & 0.33 & $\ldots$ & 1 & 0.33 & $\ldots$ & 1 & 0.46 & $\ldots$ & 1 \\
\hline \multirow{2}{*}{ Element $^{a}$} & \multicolumn{3}{|c|}{ S-138 } & \multicolumn{3}{|c|}{ S-290 } & \multicolumn{3}{|c|}{ S-364 } & \multicolumn{2}{|c|}{ NGC 5897} & \\
\hline & {$[\mathrm{X} / \mathrm{Fe}]$} & $\sigma$ & $N$ & {$[\mathrm{X} / \mathrm{Fe}]$} & $\sigma$ & $N$ & {$[\mathrm{X} / \mathrm{Fe}]$} & $\sigma$ & $N$ & $\langle[\mathrm{X} / \mathrm{Fe}]\rangle$ & $\sigma$ & \\
\hline $\mathrm{Fe}_{\mathrm{CaT}}^{b}$ & -1.97 & $\ldots$ & $\ldots$ & -2.00 & $\ldots$ & $\ldots$ & -1.97 & $\ldots$ & $\ldots$ & -1.94 & 0.05 & \\
\hline $\mathrm{Fe}_{\mathrm{MgI}}^{c^{c} \mathrm{I}}$ & -1.74 & $\ldots$ & $\ldots$ & -1.73 & $\ldots$ & $\ldots$ & -1.71 & $\ldots$ & $\ldots$ & -1.73 & 0.03 & \\
\hline $\mathrm{Fe} \mathrm{I}$ & -2.11 & 0.11 & 121 & -2.02 & 0.16 & 136 & -2.03 & 0.15 & 129 & -2.04 & 0.04 & \\
\hline Fe II & -2.01 & 0.12 & 12 & -1.99 & 0.12 & 13 & -1.98 & 0.12 & 13 & -1.92 & 0.09 & \\
\hline$A(\mathrm{Li})$ & $<-0.23$ & & 1 & $<-0.08$ & & 1 & $<-0.15$ & & 1 & & & \\
\hline OI & 0.69 & 0.04 & 2 & 0.65 & 0.15 & 2 & 0.62 & 0.12 & 2 & 0.51 & 0.19 & \\
\hline Na I ${ }^{\text {NLTE }}$ & -0.03 & 0.20 & 3 & 0.14 & 0.06 & 4 & 0.16 & 0.11 & 3 & 0.27 & 0.24 & \\
\hline Mg I & 0.58 & 0.30 & 3 & 0.46 & 0.23 & 3 & 0.38 & 0.17 & 3 & 0.46 & 0.08 & \\
\hline $\mathrm{Al} \mathrm{I}$ & & & & & & 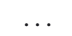 & 0.42 & 0.15 & 2 & 0.51 & 0.36 & \\
\hline Si I & 0.19 & 0.22 & 4 & 0.18 & 0.34 & 4 & 0.15 & 0.31 & 4 & 0.17 & 0.10 & \\
\hline K I & 0.76 & 0.23 & 2 & 0.60 & 0.17 & 2 & 0.62 & 0.12 & 2 & 0.55 & 0.18 & \\
\hline $\mathrm{Ca} \mathrm{I}$ & 0.43 & 0.12 & 17 & 0.38 & 0.10 & 17 & 0.37 & 0.13 & 17 & 0.39 & 0.04 & \\
\hline Sc II & 0.13 & 0.08 & 7 & 0.11 & 0.08 & 7 & 0.06 & 0.11 & 7 & 0.08 & 0.07 & \\
\hline Ti I & 0.21 & 0.21 & 17 & 0.19 & 0.17 & 17 & 0.21 & 0.15 & 17 & 0.16 & 0.04 & \\
\hline Ti II & 0.34 & 0.15 & 6 & 0.38 & 0.16 & 6 & 0.28 & 0.14 & 6 & 0.30 & 0.07 & \\
\hline V I & -0.22 & 0.06 & 2 & -0.01 & 0.04 & 2 & -0.14 & $\ldots$ & 1 & -0.14 & 0.07 & \\
\hline $\mathrm{Cr} \mathrm{I}$ & -0.25 & 0.04 & 7 & -0.19 & 0.05 & 7 & -0.24 & 0.12 & 7 & -0.20 & 0.05 & \\
\hline Mn I & -0.53 & 0.10 & 5 & -0.44 & 0.14 & 5 & -0.40 & 0.07 & 5 & -0.43 & 0.05 & \\
\hline Co I & 0.07 & 0.09 & 4 & -0.02 & 0.09 & 4 & -0.09 & 0.09 & 3 & 0.03 & 0.07 & \\
\hline $\mathrm{Ni}$ I & -0.07 & 0.23 & 25 & -0.09 & 0.23 & 24 & -0.10 & 0.25 & 25 & -0.07 & 0.02 & \\
\hline $\mathrm{Cu}$ I & -0.82 & 0.12 & 2 & -0.78 & $\ldots$ & 1 & -0.63 & $\ldots$ & 1 & -0.70 & 0.08 & \\
\hline $\mathrm{ZnI}$ & 0.08 & 0.03 & 2 & 0.01 & 0.09 & 2 & 0.03 & 0.04 & 2 & 0.07 & 0.04 & \\
\hline Y II & -0.35 & 0.11 & 5 & -0.14 & 0.12 & 5 & -0.26 & 0.14 & 5 & -0.29 & 0.11 & \\
\hline Ba II & 0.05 & 0.17 & 3 & 0.03 & 0.08 & 3 & -0.01 & 0.04 & 3 & -0.03 & 0.08 & \\
\hline La II & 0.06 & 0.19 & 4 & 0.37 & 0.18 & 4 & 0.33 & 0.13 & 4 & 0.19 & 0.14 & \\
\hline Ce II & -0.02 & $\ldots$ & 1 & -0.02 & $\ldots$ & 1 & 0.16 & $\ldots$ & 1 & -0.11 & 0.16 & \\
\hline PrII & 0.60 & $\ldots$ & 1 & & $\ldots$ & $\ldots$ & 0.46 & $\ldots$ & 1 & 0.39 & 0.20 & \\
\hline $\mathrm{Nd}$ II & 0.14 & $\ldots$ & 1 & 0.17 & $\ldots$ & 1 & 0.29 & $\ldots$ & 1 & 0.16 & 0.07 & \\
\hline Sm II & 0.31 & 0.11 & 4 & 0.32 & 0.10 & 5 & 0.39 & 0.05 & 6 & 0.31 & 0.06 & \\
\hline Eu II & 0.29 & 0.01 & 2 & 0.57 & 0.03 & 2 & 0.50 & $\ldots$ & 1 & 0.45 & 0.10 & \\
\hline Gd II & 0.51 & $\ldots$ & 1 & 0.65 & $\ldots$ & 1 & 0.61 & $\ldots$ & 1 & 0.48 & 0.12 & \\
\hline
\end{tabular}

Notes. ${ }^{(a)}$ Ionized species and $\mathrm{O}$ are given relative to Fe II. Abundance ratios are listed relative to iron, save for Fe I and Fe II (relative to $\mathrm{H}$ ) and Li. (b) Metallicity estimate based on the calcium triplet calibration of Starkenburg et al. (2010). ${ }^{(c)}$ Metallicity estimate based on the Mg I calibration of Walker et al. (2007), on the metallicity scale of Carretta \& Gratton (1997). 